\title{
Multiphase graphitisation of carbon xerogels and its dependence on their pore size
}

MARÍA CANAL-RODRÍGUEZ, LUIS A. RAMÍREZ-MONTOYA, SARA F. VILLANUEVA, SAMANTHA L. FLORES-LÓPEZ, J. ANGEL MENÉNDEZ, ANA ARENILLAS, MIGUEL A. MONTES-MORÁN*

Instituto Nacional del Carbón, INCAR-CSIC, Apartado 73, 33080 Oviedo, Spain

* Corresponding author phone: +34 985118996. E-mail: miguel.montes@csic.es (Miguel A. Montes-Morán) 


\section{Abstract}

Six carbon materials were obtained from the carbonisation of resorcinol/formaldehyde xerogels. All carbon xerogels (CXs) showed essentially the same microporosity but differed in their meso- or macroporosity, covering a wide interval of average meso- or macropore sizes from $10 \mathrm{~nm}$ to $3000 \mathrm{~nm}$. The graphitisation of the CXs was heterogeneous, as detected by X-ray diffraction. The relative amount of the amorphous, turbostratic and graphitic carbon phases on the graphitised xerogels was different depending on the pore size of the CXs. Crystalline parameters such as interlayer spacings $\left(d_{002}\right)$ and crystallite sizes along the c-axis $\left(L_{c}\right)$ were calculated from the different contributions and were also found to depend on the pore size of the parent CXs. Transmission electron microscopy and Raman spectroscopy analyses helped to identify nanostructures that could be assigned to the three carbon components of the graphitic xerogels. The occurrence of most of these nanostructures was compatible with a solid-phase transformation of the amorphous precursor. The electrical conductivity of the graphitised xerogels also depended on their original pore size, with values ranging from $2 \mathrm{~S} \mathrm{~cm}^{-1}$ for the materials with a $10 \mathrm{~nm}$ pore size to $18 \mathrm{~S} \mathrm{~cm}^{-1}$ for the materials with bigger pore sizes. 


\section{Introduction}

The structural evolution of carbons at high temperatures is arguably one of the most explored aspects in Carbon Science since the original works of Rosalind Franklin [1-3]. Yet, a precise understanding of the structure of the non-graphitising carbons is still a matter of discussion and interest [4-6]. This paper provides some new findings that could be of help in that particular quest.

On the basis of XRD studies, Franklin suggested the occurrence of the so-called multiphase (or heterogeneous) graphitisation for a number of non-graphitising carbons when heated between $2000^{\circ} \mathrm{C}$ and $3000^{\circ} \mathrm{C}$. The (002) band of these high temperature treated carbons showed a convoluted profile with two or even three contributions to the overall signal [1-3]. These contributions revealed that even though those carbons were essentially amorphous, crystalline structures were also present. Further studies demonstrated that those two or three contributions became more evident in experiments regarding the catalytic graphitisation of non-graphitising carbons by different metals [7-10]. More relevant for the present study, heterogeneous graphitisation was also clearly observed in XRD analyses of high temperature treated non-graphitising precursors that were heavily ground before the heat treatment, in absence of any catalyst $[11,8]$. Thus, in addition to the amorphous (A-carbon) contribution, two other bands were found to contribute to the (002) profile of those materials, one located at ca. $26^{\circ} 2 \theta$ (using CuKa radiation) that would correspond to carbon crystallites with an interplanar spacing of ca. $0.340 \mathrm{~nm}$ (turbostratic or Tcarbon), the other corresponding to approx. the $d_{002}$ of hexagonal graphite, i.e., 0.335 $\mathrm{nm}$ (graphitic or G-carbon). Further studies explored additional effects on the heterogeneous graphitisation of non-graphitising carbons. Carbons derived from thermosetting resins (mainly phenolic resins) were systematically select to carry out 
these studies for a number of reasons, including the technological relevance of glassy carbons [12,13]. Among others, the effect of specific phenolic monomers on the multiphase graphitisation of resin-derived carbons was appraised [14-16].

In addition to XRD, TEM analysis was soon a fundamental tool to elucidate the structure of high temperature treated carbons at the molecular level $[17,18]$. Different structural models were then proposed from TEM images of non-graphitising carbons treated at temperatures as high as $3000^{\circ} \mathrm{C}$ [18-21]. Still, the link between XRD studies describing the coexistence of $\mathrm{A}-$, $\mathrm{T}$ - and G-carbon phases in these carbons and their TEM analysis is somehow elusive [21-23]. It must be mentioned at this point the relatively recent findings related to the solid-phase transformation of non-graphitising carbons based on the works of Harris [24-27]. According to experimental evidences, $\mathrm{sp}^{2}$-ordered structures can self-assemble on the surface of non-graphitising precursors if enough energy for the phase transformation is provided, in absence of catalysts [28]. This model relies on the formation, early in the growth process, of carbon nanostructures with adequate geometries that will act as seed structures of the selfassembly process. The model, which describes reasonably well the growth of fullerenes and nanotubes $[27,28]$, could also explain the occurrence of T- and Gcarbon regions in non-graphitising carbons [28]. Actually, molecular-dynamic calculations have shown that graphite-like domains of $\mathrm{sp}^{2}$-bonded sheets result when modelling the evolution of amorphous carbon structures with temperature if the model includes two periodic (2D) boundaries. The experimental equivalent of a 2D periodicity would correspond to the growth of carbon thin films [28]. Moreover, the occurrence of graphite polyhedral crystals on glassy carbons would also support the growth mechanism of highly ordered carbons on highly disordered precursors treated at high temperatures [29]. 
Carbon xerogels (CXs) prepared from resorcinol $(R)$ and formaldehyde (F) organic gels (OXs) are non-graphitising carbons presenting bi-modal micro-, and meso- or macroporous size distributions. The tight control of a number of synthesis variables, including the $\mathrm{R} / \mathrm{F}$ ratio, dilution of the reactants on the solvent $(\mathrm{D}), \mathrm{pH}$ and methanol content of the solution, brings about a precise control of the meso- or macroporosity of the resulting OXs $[30,31]$. Carbonisation of these OXs renders CXs that preserve in essence the meso- or macroporosity of the parent OXs while developing the micropores due to volatiles emission. This offers a family of highly pure carbons with meso- or macroporosities that span the entire nanoscale (from $2 \mathrm{~nm}$ to $5000 \mathrm{~nm}$ ) [32].

All these properties make CXs a very interesting family of carbons to study their behaviour at high temperatures. Specifically, the effect of the porosity on the heterogeneous graphitisation of these materials may contribute to a better understanding of this complex phenomenon on non-graphitising carbons.

\section{Experimental}

\subsection{Materials}

Organic xerogels (OXs) were synthesised from resorcinol (Indspec, 99.6\% purity)/formaldehyde (Merck, 37\% aqueous solution, with $0.7 \%$ methanol) (RF) mixtures in deionised water. The microwave-assisted synthesis of these resorcinol/formaldehyde organic xerogels has been profusely reported in previous works [10,30-33]. Tight control over the pore development on these materials is attained by tuning four synthesis variables, namely the $R / F$ ratio, the dilution ratio $D$ (defined as the solvent/reactants molar ratio, see [30]), the $\mathrm{pH}$ of the sol and the amount of $\mathrm{MeOH}$ of the formaldehyde solution ( $\mathrm{MeOH} \%)$. The recipes selected for the preparation of the six OXs are shown in Table $1 . \mathrm{MeOH} \%$ was $0.7 \%$ in all cases. 
Numerals on sample labels correspond to the meso- or macropore rounded average size (in nm) of the OXs.

Carbon xerogels (CXs) were prepared from the OXs by heating them $(25 \mathrm{~g})$ in a quartz reactor (i.d. $30 \mathrm{~mm}$ ) placed in a horizontal electrical tube furnace (Carbolite Type MFT $12 / 38 / 400$ ), under a nitrogen flow rate of $300 \mathrm{ml} \mathrm{min}^{-1}$ up to $1000{ }^{\circ} \mathrm{C}$ at $50{ }^{\circ} \mathrm{C} \mathrm{min}^{-1}$ and $2 \mathrm{~h}$ dwell time.

High temperature treatments of CXs were carried out in a graphite furnace (Xerion Advanced Heating, Germany). $1 \mathrm{~g}$ of $2-4 \mathrm{~mm}$ CX particles was placed in a graphite crucible and heated under $\operatorname{Ar}\left(2 \mathrm{~L} \mathrm{~min}^{-1}\right)$ up to the selected temperature at heating rates of $50 \stackrel{\circ}{\circ} \mathrm{min}^{-1}$ from room temperature to $1000 \stackrel{\circ}{\circ}$; $25^{\circ} \mathrm{C} \mathrm{min}^{-1}$ from $1000-2000 \stackrel{\circ}{\circ}$; and $10 \stackrel{\circ}{\circ} \mathrm{min}^{-1}$ from $2000-2800^{\circ} \mathrm{C}$. Dwelling times at the maximum temperature of treatment were $2 \mathrm{~h}$. High temperature treated samples are labelled as "graphitised xerogels" (GXs). Unless otherwise stated in the paper, GX nomenclature will be used to refer to the CXs treated at $2800^{\circ} \mathrm{C}$. Samples treated at lower temperatures (i.e., $\left.2000^{\circ} \mathrm{C}\right)$ will be referred as CX-pore size-2000.

In addition to the graphitisation of particulates, selected CXs samples were ground using a agate hand mortar down to $<25 \mu \mathrm{m}$ particle size and heat treated in the graphite oven as just detailed.

A glassy carbon (Sigradur ${ }^{\circledR}$ rod, from HTW GmbH) and graphite (TIMREX® KS75, from TIMCAL) were also used for comparative purposes.

\subsection{Characterisation of the materials}

Helium densities $\left(\rho_{\mathrm{He}}\right)$ were determined in an AccuPyc 1330 pycnometer (Micromeritics), after outgassing the samples at $120 \stackrel{\circ}{\mathrm{C}}$ overnight. Textural properties 
of the materials were measured using $\mathrm{Hg}$ intrusion (AutoPore IV porosimeter, also from Micromeritics), due to their meso- or macroporous nature. $\mathrm{Hg}$ densities $\left(\rho_{\mathrm{Hg}}\right)$, pore size distributions and open porosities (s, see eq. 1) were determined from the $\mathrm{Hg}$ intrusion data up to a maximum operating pressure of $227 \mathrm{MPa}$. Prior measurements, samples were outgassed at $120 \stackrel{\circ}{C}$ overnight.

$\mathrm{S}=\left[1-\left(\rho_{\mathrm{Hg}} / \rho_{\mathrm{He}}\right)\right] \times 100$

In addition to $\mathrm{Hg}$ porosimetry, $\mathrm{N}_{2}$ adsorption-desorption isotherms performed at $-196{ }^{\circ} \mathrm{C}$ (Tristar II, Micromeritics) were also carried out after using similar outgassing conditions as described above. In spite of the limitation of the technique for describing the porosity of the samples, several textural parameters $\left(S_{\mathrm{BET}}\right.$, micropore volumes, etc.) were calculated from adequate models.

XRD measurements were carried out in a D8 Advance diffractometer (Bruker) equipped with a CuK ${ }_{\alpha}$ X-ray source operated at $40 \mathrm{kV}$ and $40 \mathrm{~mA}$. Reflected rays were collected using a Göbel mirror configuration. Scans were performed between $10-90^{\circ} 2 \theta$ at $0.02^{\circ} 2 \theta$ steps with acquisition times of $5 \mathrm{~s} \mathrm{step}^{-1}$. Fitting of the (002) bands (12-38응 $2 \theta$ ) of the GXs was performed with a commercial software (Casa Software Ltd.) using two or three Gaussian peaks and a linear background. All representative parameters of the Gaussian peaks were allowed to float during the fitting. An instrumental broadening $0.16^{\circ} 2 \theta$ was obtained for an internal standard of silicon and was used for the correction of the FWHM values.

SEM images were obtained in a Quanta FEG 650 microscope using an accelerating voltage of $25 \mathrm{kV}$ and an Everhart-Thornley secondary electron detector (ETD). Samples were attached to an aluminium tap using conductive double-sided adhesive tape. No further coating was used. 
HR-TEM analyses were performed in a JEOL JEM-2100F microscope operated at an accelerating voltage of $200 \mathrm{kV}$ and equipped with a field emission gun and an ultrahigh resolution pole-piece. This microscope has a point resolution $>0.19 \mathrm{~nm}$. Samples were dispersed in ethanol, sonicated, sprayed onto a carbon-coated copper grid and then allowed to air-dry.

Raman spectroscopy was carried out on a LabRAM HR 800 Horiba Jobin Yvon spectrometer equipped with a CCD detector. A green laser $(\lambda=532 \mathrm{~nm})$ was used as incident beam ( $2 \mu \mathrm{m}$ in diameter), which was focused onto the sample surface using an optical microscope (BXFM, from Olympus; 100x objective lenses) coupled to the spectrometer. A 1800 lines $\mathrm{mm}^{-1}$ grating was used to split the incoming radiation, the spatial resolution of the Raman measurements being of $0.3 \mathrm{~cm}^{-1}$. Typical exposure times of 20 s were used in each of two consecutive spectrum acquisitions.

The electrical conductivity of the samples was determined using a four-point probe (FPP) apparatus (SR-4-6L model from Everbeing Intl. Co.), as detailed elsewhere $[34,10]$. Briefly, thin pellets were prepared from powdered samples $(<75 \mu \mathrm{m})$ using polytetrafluoroethylene (PTFE) as binder. Pellets were then tested in the FPP equipment by carefully placing the FFP on the pellet surface. Pellets resistivity was finally measured using the Van der Pauw method [35]. For a given sample, at least 4 pellets were prepared and at least 6 measurements in each pellet were carried out to obtain its average conductivity value. Pellet thickness was carefully controlled (120-180 $\mu \mathrm{m})$ to minimise possible sample to sample deviations. 


\section{Results and discussion}

\subsection{Heterogeneous graphitisation of CXs}

Figure 1 shows the pore size distributions (PSDs) of the six CXs and their GXs counterparts, as determined from $\mathrm{Hg}$ intrusion. In general, PSDs are relatively narrow, especially those of $\mathrm{CX}-10$ and $\mathrm{CX}-100$. Tight control of pore size becomes more difficult as the average pore size increases, resulting in wider PSDs [28]. In terms of porosity values (s), the CXs can be grouped in two different sets, CX-10 on one hand, which is a less porous, highly dense carbon material $(s=55 \%)$; and the rest of CXs on the other, with porosity values ranging between $74-87 \%$. As a consequence, CX-10 looked more like a glassy material than the rest of CXs.

Graphitisation of CXs tends to reduce to a different extent the maximum of the PSDs of the materials. In addition, graphitisation diminishes the pore volume of the samples as measured by this technique $\left(\mathrm{V}_{\mathrm{Hg}}\right.$, Table 2). This pore size and/or pore volume reduction after the high temperature treatment brings about denser $\left(\rho_{\mathrm{Hg}}\right)$, less porous (s) materials (Table 2). Nonetheless, $\rho_{\mathrm{Hg}}$ of $\mathrm{GX}-10\left(1.037 \mathrm{~g} \mathrm{~cm}^{-3}\right)$ almost doubles the average value of the rest of GXs (around $0.5 \mathrm{~g} \mathrm{~cm}^{-3}$ ).

Whereas $\mathrm{Hg}$ porosimetry reveals the shift of the PSDs of the CXs towards lower pore size values (except for $\mathrm{CX}-100$ ), $\mathrm{N}_{2}$ adsorption/desorption isotherms point out the collapse of the smaller pores after the high temperature treatment (Figure SI1). CXs have a significant amount of micropores with $V_{D R}$ ranging between $0.20-0.27 \mathrm{~cm}^{3} \mathrm{~g}^{-1}$ for all materials (Table 2). These values decrease dramatically (below $0.05 \mathrm{~cm}^{3} \mathrm{~g}^{-1}$ ) after graphitisation. Actually, microporosity on GX-700, -1000 and -3000 is hardly detected. Mesopore volumes $\left(\mathrm{V}_{\text {meso, }}\right.$, Table 2) calculated from the nitrogen isotherms match 
reasonably well those given by $\mathrm{Hg}$ intrusion $\left(\mathrm{V}_{\mathrm{Hg}}\right.$, also Table 2$)$ only for $\mathrm{CX}-10$ and $\mathrm{GX}$ 10 , as expected [36].

As mentioned in the Introduction, carbon xerogels based on R/F precursors are nongraphitising carbons that show very limited crystalline order after high temperature treatments (Figure SI2). The diffraction pattern of sample GX-100 shown in Figure SI2 already shows a composite (002) reflection. However, the multiphase graphitisation of the CXs under study was very different, as measured by XRD (Figure 2), with the A-, Tand G- relative contributions to the (002) band depending on the pore size of the original CXs. The XRD profile of the GX-10 resembles that of a glassy carbon (see Figure SI3). The composite (002) profiles of the GXs with pore sizes $\geq 350 \mathrm{~nm}$ (Figure $2 b)$ are very rarely observed in the heterogeneous graphitisation of other nongraphitising precursors. They are, however, characteristic of the catalytic graphitisation of a number of precursors including phenol/formaldehyde resins $[7,8]$, and RF resins $[9,10]$. At first sight (Figure 2), it seems that the amount/quality of the crystalline phases of the GXs increases as the pore size increases. Additional confirmation of such increase is the incipient (004) reflection noticed in the GX-700, GX-1000 and GX-3000 XRD patterns at ca. $54.5^{\circ} 2 \theta$ (see insets of Figure $2 b$ ), as well as the widening of the (10) band $\left(40-47^{\circ} 2 \theta\right)$. It is finally worth noticing the doublet of the $(004)$ reflection of the XRD patterns of the GXs of bigger pore size, that should correspond to the T-and G-carbon contributions.

In order to quantify the differences in the multiphase graphitisation of the GXs, deconvolution of the (002) bands was carried out. Gaussian peaks were thus used to fit the experimental data, by iterative searching of all relevant peak parameters (see experimental section). Three contributions to the overall (002) profile were assumed for all GXs except GX-10 and GX-100, for which the T-contribution was hardly noticed and was thus not considered (Figure SI4). To simplify the notation and avoid confusion, the 
conventional amorphous A-contribution has been named here "Non-graphitic", since in the case of the two GXs having the lowest pore sizes this "Non-graphitic" contribution is assumed to include T-carbon. Figure $3 a$ shows the fractional contribution of the different carbon phases to the (002) profile, expressed as percentages of the total area of the (002) diffraction band. According to this semi-quantitative analysis, the amount of G-carbon increases from $\mathrm{GX}-10$ to $\mathrm{GX}-100$. This amount remains then fairly constant for GXs with bigger pores. The analysis of the T-carbon contribution is restricted to GXs with average pore sizes $\geq 350 \mathrm{~nm}$, as just mentioned. For those four materials, the T-contribution decreases as the pore size of the parent $\mathrm{CX}$ increases.

Crystalline parameters were calculated from the peak maxima and FWHMs of the three contributions using Bragg and Scherrer equations, respectively (Table 3). Results of Table 3 indicate that the meso- or macroporous size of the original CXs affects the quality of the G-carbon of the resulting GXs. Thus values of interplanar spacings of GX1000 and GX-3000 were similar to that measured for the commercial graphite, whereas the $L_{c}$ value of $G X-3000(40.3 \mathrm{~nm})$ is also very close to that of the graphite $(51 \mathrm{~nm}) . L_{c}$ of the G-carbon contribution improves as the pore size of CX increases above $100 \mathrm{~nm}$ (Figure 4). The crystallinity of the turbostratic, and especially the non-graphitic fraction is very similar for all GXs. Typical $d_{002}$ values of ca. $0.340 \mathrm{~nm}$ were calculated for $\mathrm{T}$ carbon contributions of the GXs, with $L_{c}$ values ranging from 9-11 nm.

Calculation of $L_{a}$ values for the GXs was carried out using the (10) band for all materials. Although qualitative differences between the (10) bands of the GXs have been already mentioned (i.e., the incipient splitting of the band observed in the profiles of GX-700, -1000 and -3000 , insets of Figure 2), the quality of the signal was, in any case, not good enough to perform a sound deconvolution of the (10) profile. As a consequence, $L_{a}$ values depicted in Table 3 are relatively small and similar to that of the glassy carbon, for all GXs. 
Different reasons could explain the effect of the pore size on the multiphase graphitisation of the CXs. The possibility of different pore sizes affecting the thermal diffusion during the heat treatment of the CXs was discarded after performing a graphitisation experiment of $\mathrm{CX}-100$ using a much lower heating rate $\left(5^{\circ} \mathrm{C} \mathrm{min}^{-1}\right)$ from $1000-2800 \stackrel{\circ}{ } \mathrm{C}$ (see experimental section). The XRD patterns of both $\mathrm{GX}-100$ samples were almost identical (Figure SI5). On the other hand, the possibility of chemical differences between OXs determining their different behaviour in the multiphase graphitisation [16], was also discarded. So far, we have not found any experimental evidence of such chemical differences between organic xerogels of different pore size [37]. The different multiphase graphitisation of the CXs is thus ascribed to differences on their nanostructure.

The nanostructure of RF-based carbon xerogels have been intensely characterised since the seminal work of Pekala [38]. As mentioned in the Introduction, the clusters resulting from the RF polymerisation remain as the basic structural units of the CXs after carbonisation. The pore size distributions, as measured by mercury intrusion (Figure 1), correspond to inter-clusters voids, which depend on both the clusters size and the clusters spatial arrangement (cluster crosslinking) [30-33,39,40]. Although a cluster size distribution of the carbon xerogels is not provided here, it can be fairly assumed that the average cluster size of the CXs synthesised in this work increases from CX-10 to CX-3000 (Figure 5). This assumption can be safely made for the CX-10, $-700,-1000$ and -3000 , i.e., the average cluster size of $C X-10<<C X-700<C X-1000<$ CX-3000. For these four CXs, the synthesis conditions of the OXs counterparts were the same except for the $\mathrm{pH}$ of the initial solutions (Table 1). The effect of this particular variable on the $\mathrm{CX}$ cluster sizes has been already studied in detail, the lower the $\mathrm{pH}$ the higher the average cluster size $[32,33,39,40]$. For CX-100 and CX-350, several synthesis variables were altered with respect to the rest of CXs (Table 1), which most 
likely resulted in average pore sizes bigger than expected if only the solution $\mathrm{pH}$ were altered. Still, the average pore size of CX-100 is an order of magnitude higher than CX10, a big enough difference to guarantee that the average cluster size of the CX-100 should be bigger than that of $\mathrm{CX}-10$, under the $\mathrm{pH}$ conditions used (Figure 5).

In short, and for the six CXs under study, i) it is reasonable to assume that CXs with higher (measured) average pore sizes will have higher average cluster size, and ii) it is suggested that the different multiphase graphitisation behaviour depends on the average cluster size of the parent CXs. It should be clear that this does not mean that there exists a direct relationship between average pore and cluster size for this series of CXs.

Also relevant to the influence of the cluster size on the multiphase graphitisation of GXs is the effect of the macroscopic particle size of the CXs. It has been demonstrated that the grinding of phenol/formaldehyde resins prior to their high temperature treatment changes dramatically the (002) reflections of the resulting materials, from a broad band (similar to that of $\mathrm{GX}-10$ ) to a composite profile with two sharp peaks corresponding to the T- and G-carbon contributions [11,22]. It was thus decided to carry out graphitisation experiments with selected CXs ground down to a particle size below 25 $\mu \mathrm{m}$. The (002) profiles of the resulting $G X s<25$ are shown in Figure 6a. These diffraction patterns are very different from those of Figure 3 for all materials. A similar behaviour than that described in [11] is observed, i.e., the splitting of the T-and Gcarbon contributions became much more evident after grinding of the non-graphitising precursor. However, there is again a clear difference between the different $G X s<25$. The relative proportions of the three contributions to the (002) profile are shown in Figure 3b. After grinding, the Non-graphitic contribution decreases significantly whereas the G-carbon fraction almost doubled. This increase of the G-carbon is also confirmed when comparing the (10) bands of the GXs and GXs<25 (Figure 6b). The 
(10) band of the GXs $<25$ shows a well-defined peak at $42.54^{\circ} 2 \theta$ which correspond to the $(100)$ reflection of graphite $\left(d_{100}=0.212 \mathrm{~nm}\right)$. As in the case of the particulate GXs, the relative areas of the G-carbon contribution remain fairly constant for all GXs $<25$ (Figure 3b). However, the T-carbon contribution to the (002) profiles of the GXs<25 of bigger pore sizes ( $\geq 350 \mathrm{~nm}$ ) does not decrease as the pore size increases (Figure 3b). The most pertinent observation regarding this T-component in $G X s<25$ is the sharpness of the contribution, especially in the case of GX-1000<25 and GX-3000<25 (Figure 6a).

Interplanar spacing $\left(\mathrm{d}_{002}\right)$ values of the G-, T- and Non graphitic-carbon fractions were 0.336-0.337 nm, 0.342-0.343 $\mathrm{nm}$ and $0.347-0.348 \mathrm{~nm}$, respectively for all $\mathrm{GXs}<25 . \mathrm{L}_{\mathrm{c}}$ values of the different contributions are also plotted in Figure 4 (empty symbols). It should be noticed that it has been assumed that the grinding process did not affect the macroporosity of the CXs. The mercury intrusion technique is limited to particulate $(\mathrm{mm})$ samples and results obtained in powdered samples are meaningless. In any case, whereas the assumption of unaltered macroporosity might be questioned for $\mathrm{CX}$ 1000 and CX-3000, it should stand for the other two CXs. Results of Figure 4 corresponding to the $G X s<25$ are quite intriguing, with $L_{c}$ values for the graphitic contribution almost constant for all GXs<25 (ca. $27 \mathrm{~nm}$ ), significantly lower than the ca. $40 \mathrm{~nm}$ obtained for the particulate GXs with big pores (Table 3 and Figure 4). On the other hand, the evolution of the $L_{c}$ of the T-contribution with $G X s<25$ pore size resembles that of the G-contribution of the particulate GXs, with a monotonic rise as the pore size increases above $100 \mathrm{~nm}$, to the point of T-carbon reflections of the GX$3000<25$ rendering c-stacking sizes as high as the commercial graphite (Table 3).

\subsection{G-, $T$ - and A-nanostructures}

The set of different GXs (including $G X s<25$ ) was further analysed to explore the possibility of identifying the nanostructures that could be ascribed to their (002) G-, T- 
and A-contributions. According to the XRD profile analysis, all G-, T- and Ananostructures were expected to occur in all GXs. TEM analysis confirmed this and images were thus selected to show different nanostructures that were representative of all GXs. In that sense, images ascribed to a particular GX in the following Figures are qualitative examples that were also found in the rest of GXs.

Starting with the amorphous carbon, HRTEM analysis confirmed the presence of two types of nanostructures (Figure 7). Figure 7a shows an example of a highly disordered region which resembles the nanostructure of non-graphitising carbons obtained at relatively low temperatures. For example, this type of images was predominant in CXs treated up to $2000{ }^{\circ} \mathrm{C}$ (see Figure SI6) [13], and would indicate that significant parts of the CXs clusters evolve very little with temperature. They would strongly contribute to the widening of the (002) of the GXs towards lower $2 \theta$ values (see Figure SI2 for the evolution of the XRD profile of a CX with temperature). Figure $7 \mathrm{~b}$ shows a different type of nanostructures where some local order exist, with fringes running parallel but showing strong curvatures. These nanostructures were ubiquitous in all GXs TEM analysis and have been already described and discussed for different high temperature treated carbon materials including glassy carbons and carbon blacks [18,22-26]. Electron diffraction carried out on the curved graphene area (inset of Figure $7 \mathrm{~b}$ ) shows $d_{002}$ values that would also contribute to the Non-graphitic fraction of the GXs (Table 3). Evidence of closed shells nanostructures were also found in the GXs (see Figure SI7). Although it is not very relevant for the current discussion, it should be mentioned that those closed shells were not empty, with some ordered textures being observed inside the shell (despite the low quality image) (Figure SI6, bottom).

The formation of the curly structures of Figure $7 \mathrm{~b}$ has been well described by a solidphase graphitisation mechanism [24,28]. According to molecular dynamic calculations, these curved graphene arrangements cannot form in absence of voids (pores). Since 
all CXs have very similar micropore volumes (Table 2), it is thus expected that all GXs would end with similar relative amounts of Non-graphitic contribution. In other words, the meso- or macropore size of the original CXs would have little effect in the development of the non-graphitic contribution to the (002) diffraction band, as it seems to be the case (Figure 3) (the non-graphitic carbon contribution of GX-10 and GX-100 in Figure 3a may be overestimated by an eventual T-contribution).

The previous conclusion links the microporosity of the original CXs clusters with the development of non-graphitic structures in GXs shown in Figure 7b. However, the way that microporosity evolves with temperature might also condition the formation of those nanostructures. In a very recent paper [3], it has been shown the concomitant evolution of micropores and carbon nanostructure in phenolic resins treated at high temperatures (up to $3000^{\circ} \mathrm{C}$ ). By using small-angle neutron scattering (SANS) analysis, the authors follow the development of closed or inaccessible porosity, showing the enlargement of the initial microporosity as the carbon nanostrucutres of the pore walls grow in size. In the case of the CXs under study, it seems clear from results of Table 2 that GXs contain significant amounts of closed pores. Indeed, He densities of the GXs are lower than those of the CXs counterparts, even though $\mathrm{Hg}$ intrusion measurements point out the densification of the materials after graphitisation. This suggests that the access of He to the microporosity of the CXs is restricted after the high temperature treatment, possibly by the collapse of the micropore entrances. The relevant point for this discussion is that all particulate GXs end up with very similar He densities (Table 2), with a closed porosity in the GXs clusters amounting ca. 25\% (assuming a $\rho_{\mathrm{He}}$ of $2 \mathrm{~g}$ $\mathrm{cm}^{-3}$ for graphite). This would suggest that the clusters microporosity would be evolving in a similar fashion regardless the original cluster size.

As for the two types of ordered carbon, T-carbon and G-carbon, present in the GXs, TEM evidences were puzzling. Areas showing highly ordered (parallel) graphene 
stacking were found in all GXs, including GX-10 (Figure 8a). Measurement of spacing between lattice fringes rendered $d_{002}$ values that could well correspond to turbostratic $(\geq 0.34 \mathrm{~nm})$ or graphitic $(<0.34)$ areas (Figures $8 a-8 c$ and $8 e)$. Although this type of parallel graphene arrangements has been ascribed to the G-carbon contribution [21], they have been also identified as T-carbon phase [8]. Finally, flake-like areas of most likely graphitic carbon were found in particulate GXs of bigger ( $\geq 750 \mathrm{~nm})$ pore sizes (Figure SI8).

There are several questions that need to be addressed regarding their G- and T-carbon contributions from the XRD analysis of the GXs and their dependence on the pore size of the materials (Figures 3 and 4). It is proposed here that a solid-state graphitisation model may be useful to understand at least some of them. The original "seed structures" in this case would be areas of carbonised RF polymer chains as much long and well oriented as possible. The seeds would render T-carbon or G-carbon nanostructures depending on the alignment of the RF polymeric chains and/or the degree of aromatisation reached during their carbonisation. Also according to this model, the growth of the graphene parallel layers would occur mainly on the outermost surfaces of the clusters and the self-assemble of $\mathrm{sp}^{2}$ ordered sheets proceeds from the clusters surfaces into their interior (Figure 8d). The Lc values of both T- and Gcontributions would be an approximation of the "depth" of the solid phase transformation. Considering the Lc values of the T- and G-contributions of the particulate GXs (Table 3 and Figure 4), the model would explain the progressive increase of the non-graphitic contribution from GX-350 to GX-3000 shown in Figure 3a. Thus, the relative contribution of the "crystalline" shell would decrease as the cluster size increase. In the case of those four GXs, the increase of the Non-graphitic contribution is attained at the expense of the T-contribution (Figure 3a). 
The same solid-phase graphitisation model would also explain the improvement of the G-carbon quality as the pore size increases (Figure 4). For a given cluster thickness, the geometrical curvature of the clusters with micron sizes will be much lower than that of small clusters. It would be then simpler for graphene layers to stack in a parallel, more perfect way as the curvature of the surface decreases. The progressive graphitisation would have more space to stack perfectly aligned graphene sheets before starting the distortion by curving. Hence, G-carbon structures approaching perfect graphite crystallites would be more likely found in GXs with big clusters (Table $3)$.

Grinding the particulate CXs down to $25 \mu \mathrm{m}$ particle size prior graphitisation would alter the multiphase graphitisation by increasing the external surface area available for solidstate transformations, mainly in $\mathrm{GX}-1000<25$ and $\mathrm{GX}-3000<25$. This seems to stir the growth of big-size crystallites of T-carbon in the two $\mathrm{GXs}<25$ of bigger pore sizes (Figure 4, Figure 8e). Why this grinding process favours the quality of the T-carbon contribution and not that of the G-contribution remains as an open question.

The final part of this section is devoted to the presence of carbon or graphitic whiskers in the GXs. Evidences of these graphitic whiskers are shown in Figures 9 and 10. Although they were especially profuse in GXs<25 (Figure 9b), they were eventually spotted in particulate samples (Figure 9a). The nanostructure of these graphitic whiskers resembles that of the stacked-cup carbon nanofibers [41-44]. The curvature of the graphene layers in these graphite whiskers, as observed in Figure 10b and 10c, is expected to increase the (002) interlayer spacing and the band widening with respect to ideal graphite [45].

The presence of carbon whiskers on the outer surface of the particulate GXs was also confirmed by Raman spectroscopy. Although Raman spectroscopy is also a very 
helpful tool to spot changes in the structural order of carbon materials, in this case results obtained were very limited due to the heterogeneous graphitisation of the GXs. Thus, a statistical analysis was intended to calculate representative Raman parameters (21 measurements at different spots) on the particulate GXs. For these measurements, GXs millimetre particles were ground and the resulting powders were compacted using glass slides. While measuring, it was found that the Raman spectrum was very dependent on the particular spot selected. Thus, in addition to Raman profiles characteristic of relatively disordered carbons, Raman spectra characteristic of highly ordered carbon materials were eventually found (Figure 11a and b, respectively). This was a common outcome for all GXs analysed, irrespective of their pore size. Surprisingly, the second order Raman bands of these highly ordered spots were abnormally intense (notice the different right and left intensity scales of Figure 11b). Although unusually strong second order bands have been also reported when studying graphite polyhedral crystals found in pores of glassy carbon [29], this Raman spectrum is characteristic of carbon (graphite) whiskers [46]. This prompted us to perform an additional Raman analysis of the GXs using the original 2-4 mm particles instead of the ground samples. When focusing the laser beam on the particles surface of several GXs, Raman spectra similar to Figure $11 \mathrm{~b}$ were almost exclusively obtained. This was an indication that graphite whiskers were highly concentrated on the outermost surface of the GX millimetre particles, as in the case of graphite polyhedral crystals in glassy carbons [29].

The origin of graphitic whiskers is believed to follow the so-called cone-helix growth mechanism [43]. The fact that they were especially abundant in the GXs $<25$ might be related to debris present after heavy milling of the CXs. In any case, the contribution of these particular nanostructures to the XRD patterns of the GXs and/or GXs $<25$ remains unclear. 


\subsection{Electrical conductivity of GXs}

This final section of the paper is devoted to the characterisation of the electrical conductivity of the particulate GXs, due to the general interest of carbons for electrochemical applications. Results are shown in Figure 12. As expected, the graphitisation treatment improves the electrical conductivity of the original CXs but that improvement depends strongly on the pore size of the CX. Thus, the electrical conductivity of GX-10 doubles that of the $\mathrm{CX}-10$ and it is slightly higher than that of the glassy carbon. For the rest of CXs, a massive increase of the electrical conductivity is attained after graphitisation. Values of the electrical conductivities do not change significantly for GXs of pore sizes between $350-3000 \mathrm{~nm}$, with average values ranging between 14 and $18 \mathrm{~S} \mathrm{~cm}^{-1}$. This trend suggests that conductivity in GXs could be mainly controlled by the amount of graphitic carbon present in the materials (Figure 4). However, electrical conductivity in these carbons is considered to depend on a number of factors including nanostructural perfection, particle size (see below) and porosity. In that sense, it may well happen that more than a single contribution controls the conductivity values of the particulate GXs. Electrical conductivity measurements were also carried out using GXs $<25$. Again, similar values for $\mathrm{GXs}$ with pore sizes $\geq 100 \mathrm{~nm}$ were found (Figure 12). There is a significant reduction with respect to particulate GXs, which was ascribed to the use of smaller particle size in the FPP measurements, i.e., $25 \mu \mathrm{m}$ vs $75 \mu \mathrm{m}$ for GXs $<25$ and particulate GXs, respectively. For example, the electrical conductivity of a particulate GX-100 that was tested using pellets made from powders with a particle size of $<25 \mu \mathrm{m}$ was $8.18( \pm 0.04) \mathrm{S} \mathrm{cm}^{-1}$. 


\section{Conclusions}

Resorcinol/formaldehyde CXs are non-graphitising carbons that undergo a multiphase graphitisation at high temperature $\left(2800^{\circ} \mathrm{C}\right)$. This multiphase graphitisation is characterised by the co-existence of amorphous and crystalline (which includes turbostratic and graphitic) nanostructures on the GXs. It has been demonstrated here that both the quantity and quality of the crystalline nanostructures depends on the meso- or macropore size of the original CXs, which for the studied materials was related to the size of the clusters that conform the xerogels, the higher the average pore size, the higher the cluster size. It was found that, for particulate CXs, the crystal size and interlayer stacking in the c-direction of the graphitic carbon phase, as measured by $\mathrm{XRD}$, improves significantly as the pore size of the parent $\mathrm{CX}$ increases. Analysis of the possible effect of the particle size on the heterogeneous graphitisation of the CXs was carried out by grinding the precursor down to $<25 \mu \mathrm{m}$ particle size prior graphitisation. Very different XRD profiles were obtained for these $G X s<25$, although the effect of the pore size of the original CX was still evident. Combination of microscopy techniques and Raman spectroscopy allowed us to postulate some plausible amorphous, turbostratic and graphitic nanostructures that coexist in the GXs. Experimental evidences pointed out that the presence of several of those nanostructures was compatible with a solid-phase growth mechanism, a model that ultimately helped to understand (at least partially) the effect of the pore size on the multiphase graphitisation of the CXs. Carbon or graphite whiskers were also found, especially in GXs<25 of bigger pores. Finally, the electrical conductivity of the CXs improved dramatically after graphitisation of materials with pore sizes $\geq 100 \mathrm{~nm}$. 


\section{Acknowledgments}

The authors thank the financial support received from the Ministerio de Economía, Industria y Competitividad from Spain (Project CTQ2017-87820-R) and PCTI-AsturiasFEDER (Project IDI/2018/000118). MCR also acknowledges the support from CSIC (Project I.E. 201880E010). LARM thanks CONACYT (México) for a post-doctoral grant (CVU No 330625, 2017). The authors thank Ana B. García and Amelia MartínezAlonso for providing the commercial graphite and glassy carbon samples, respectively.

\section{Supporting Information Available}

Seven figures are included as supporting information. 


\section{References}

[1] Franklin RE. Crystallite growth in graphitizing and non-graphitizing carbons. Proc Roy Soc 1951;A209:196-218.

[2] Franklin RE. The structure of graphitic carbons. Act Cryst 1951;4:253-261.

[3] Franklin RE. Homogeneous and heterogeneous graphitization of carbon. Nature 1956;177:239.

[4] Badaczewski F, Loeh MO, Pfaff T, Dobrotka S, Wallacher D, Clemens D, et al. Peering into the structural evolution of glass-like carbons derived from phenolic resin by combining small-angle neutron scattering with an advanced evaluation method for wide-angle X-ray scattering. Carbon 2019;141:169-181.

[5] Ouzilleau P, Gheribi AE, Chartrand P. The graphitization temperature threshold analyzed through a second order structural transformation. Carbon 2016;109:896-908.

[6] Shiell TB, McCulloch DG, McKenzie DR, Field MR, Haberl B, Boehler R, et al. Graphitization of glassy carbon after compression at room temperature. Phys Rev Lett 2018;120:215701.

[7] Oya A, Otani S. Catalytic graphitization of carbons by various metals. Carbon 1979;17:131-137.

[8] Oya A, Otani S. Influences of particle size of metal on catalytic graphitization of nongraphitizing carbons. Carbon 1981;19:391-400.

[9] Maldonado-Hódar FJ, Moreno-Castilla C, Rivera-Utrilla J, Hanzanawa Y, Yamada Y. Catalytic graphitization of carbon aerogels by transition metals. Langmuir 2000;16:4367-4373.

[10] Canal-Rodríguez M, Arenillas A, Menéndez JA, Beneroso D, Rey-Raap N. Carbon xerogels graphitized by microwave heating as anode materials in lithium-ion batteries. Carbon 2018;137:384-394.

[11] Kobayashi K, Shiraishi M, Suzuki K. Grinding effect on graphitization of nongraphitizing carbons. Tanso 1973;74:83-89.

[12] Kawamura K, Tsuzuku T. Partial graphitization in relation to the porosity of glassy carbon. Carbon 1974;12:352-355.

[13] Zhang Z, Brydson R, Aslam Z, Reddy S, Brown A, Westwood A, et al. Investigating the structure of non-graphitising carbons using electron energy loss spectroscopy in the transmission electron microscope. Carbon 2011;49:5049-5063.

[14] Kobayashi K, Sugawara S, Toyoda S, Honda H. An X-ray diffraction study of phenol-formaldehyde resin carbons. Carbon 1968;6:359-363.

[15] Honda H, Kobayashi K, Sugawara S. X-ray characteristics of non-graphitizing-type carbon. Carbon 1968;6:517-523. 
[16] Mitchell SJ, Pickering RS, Thomas CR. The influence of polymerization catalysts on the graphitizing behaviour of 3,5-xylenol/formaldehyde resin carbons. Carbon 1972;10:621-629.

[17] Oberlin A. Carbonization and graphitization. Carbon 1984;22:521-541.

[18] Oberlin A, Bonnamy S. A realistic approach to disordered carbons. In: Radovic LR, editor. Chemistry and Physics of Carbon. Boca Raton: Taylor\&Francis; 2013(vol 31). p. 1-83.

[19] Jenkins GM, Kawamura K, Ban LL. Formation and structure of polymeric carbons. Proc Roy Soc A 1972;327:501-517.

[20] Yoshida A, Kaburagi Y, Hishiyama Y. Microtexture and magnetoresistance of glass-like carbons. Carbon 1991;29:1107-1111.

[21] Zhang J, Shi J, Zhao Y, Guo Q, Liu L, Feng Z, et al. Structural changes in four different precursors with heat treatment at high temperature and resin carbon structural model. J Mat Sci 2012;47:5891-5899.

[22] Kasahara N, Shiraishi S, Oya A. Heterogeneous graphitization of thin carbon fiber derived from phenol-formaldehyde resin. Carbon 2002;41:1654-1656.

[23] Jurkiewicz K, Pawlyta M, Burian A. Structure of carbon materials explored by local transmission electron microscopy and global diffraction probes. C 2018;4:68.

[24] Harris PJF, Tsang SC. High-resolution electron microscopy studies of nongraphitizing carbons. Phil Mag A 1997;76:667-677

[25] Harris PJF. Structure of non-graphitising carbons. Inter Mat Rev 1997;42:206-218.

[26] Harris PJF. New perspectives on the structure of graphitic carbons. Crit Rev Sol State Mat Sci 2005;30:235-253.

[27] Harris PJF. Solid state growth mechanisms for carbon nanotubes. Carbon 2007;45:229-239.

[28] Powles RC, Marks NA, Lau DWM. Self-assembly of $\mathrm{sp}^{2}$-bonded carbon nanostructures from amorphous precursors. Phys Rev B 2009;79:075430.

[29] Gogotsi Y, Libera JA, Kalashnikov N, Yoshimura M. Graphite polyhedral crystals. Science 2000;290:317-320.

[30] Rey-Raap N, Menéndez JA, Arenillas A. RF xerogels with tailored porosity over the entire nanoscale. Mic Mes Mater 2014;195:266-275.

[31] Calvo EG, Juárez-Pérez EJ, Menéndez JA, Arenillas A. Fast microwave-assisted synthesis of tailored mesoporous carbon xerogels. J Coll Interf Sci 2011;357:541-547.

[32] Rey-Raap N, Menéndez JA, Arenillas A. Simultaneous adjustment of the main chemical variables to fine-tune the porosity of carbon xerogels. Carbon 2014;78:490499. 
[33] Rey-Raap N, Arenillas A., Menéndez JA. A visual validation of the combined effect of $\mathrm{pH}$ and dilution on the porosity of carbon xerogels. Mic Mes Mater 2016;223:89-93.

[34] Rey-Raap N, Calvo EG, Bermúdez JM, Cameán I, García AB, Menéndez JA, et al. An electrical conductivity translator for carbons. Measurement 2014;56:215-218.

[35] van der Pauw LJ. A method of measuring specific resistivity and hall effect of discs of arbitrary shape. Philips Research Reports 1958;13:1-9.

[36] Ramírez-Montoya LA, Concheso A, Alonso-Buenaposada I, García H, Menéndez JA, Arenillas A, et al. Protein adsorption and activity on carbon xerogels with narrow pore size distributions covering a wide mesoporous range. Carbon 2017;118:743-751. [37] Alonso-Buenaposada ID, Garrido L, Montes-Morán MA, Menéndez JA, Arenillas A. An underrated variable essential for tailoring the structure of xerogel: the methanol content of commercial formaldehyde solutions. J Sol-Gel Sci Tehcnol 2017;83:478-488. [38] Pekala RW. Organic aerogels from the polycondensation of resorcinol with formaldehyde. J Mat Sci 1989;24:3221-3227.

[39] Al-Muhtaseb SA, Ritter JA. Preparation and properties of resorcinol-formaldehyde organic and carbon gels. Advanced Mat 2003;15:101-114.

[40] Job N, Pirard R, Marien J, Pirard JP. Porous carbon xerogels with texture tailored by $\mathrm{pH}$ control during sol-gel process. Carbon 2004;42:619-628.

[41] Endo M, Kim YA, Hayashi T, Yanagisawa T, Muramatsu H, Ezaka M, et al. Microstructural changes induced in "stacked cup" carbon nanofibers by heat treatment". Carbon 2003;41:1941-1947.

[42] Saito Y, Arima T. Features of vapor-grown cone-shaped graphitic whiskers deposited in the cavities of wood cells. Carbon 2007;45:248-255.

[43] Dimovski S, Gogotsi Y. Graphite whiskers, cones, and polyhedral crystals. In: Gogotsi $Y$ and Presser V, editors. Carbon nanomaterials. Boca Raton: Taylor\&Francis; 2014(2nd edition). p. 89-114.

[44] Ramos A, Cameán I, García AB. Graphitization thermal treatment of carbon nanofibers. Carbon 2013;59:2-32.

[45] Li ZQ, Lu CJ, Xia ZP, Zhou Y, Luo Z. X-ray diffraction patterns of graphite and turbostratic carbon. Carbon 2007;45:1686-1695.

[46] Dong J, Shen W, Tatarchuk B. Origin of strong G' band in Raman spectra of carbon whiskers. Appl Phys Lett 2002;80:3733-3735. 
Table 1. Experimental variables used in the preparation of the organic xerogels (OXs) that were thermally treated to obtain the different graphitic xerogels (GXs).

\begin{tabular}{lcccc}
\hline & \multicolumn{4}{c}{} \\
\cline { 2 - 5 } Sample & $\mathrm{R} / \mathrm{F}$ ratio & $\mathrm{D}^{\mathrm{a}}$ & $\mathrm{pH}$ & ${\text { Yield }(\%)^{\mathrm{b}}}^{\mathrm{b}}$ \\
\hline GX-10 & 0.5 & 5.7 & 6.5 & 92 \\
GX-100 & 0.5 & 9.7 & 5.8 & 95 \\
GX-350 & 0.3 & 7.8 & 4.7 & 92 \\
GX-700 & 0.5 & 5.6 & 3.1 & 90 \\
GX-1000 & 0.5 & 5.6 & 3.0 & 93 \\
GX-3000 & 0.5 & 5.6 & 2.0 & 92 \\
\hline
\end{tabular}

${ }^{a}$ Dilution ratio (see text)

${ }^{\mathrm{b}}$ Graphitisation yield, expressed as (mass $\mathrm{GX} /$ mass $\left._{\mathrm{CX}}\right) \times 100$ 
Table 2. Textural parameters of the carbon xerogels.



${ }^{a}$ Calculated at $\mathrm{p} / \mathrm{p}_{0}=0.99$

${ }^{b} \mathrm{~V}_{\text {meso }}=\mathrm{V}_{\text {total }}-\mathrm{V}_{\mathrm{DR}}$

${ }^{\mathrm{c}} \mathrm{Hg}$ intrusion pore volume corresponding to the pore size distributions shown in Figure 1

${ }^{\mathrm{d}}$ Open porosity (eq. 1) 
Table 3. Crystalline parameters (average values) of the graphitic, turbostratic and non-graphitic contributions (see Figure 4) to the (002) XRD profile of the GXs

\begin{tabular}{lccccccc}
\hline Sample & \multicolumn{2}{c}{ G-carbon } & \multicolumn{2}{c}{ T-carbon } & \multicolumn{2}{c}{ Non-graphitic } & \\
\cline { 2 - 8 } & $\begin{array}{c}\mathrm{d}_{002} \\
(\mathrm{~nm})\end{array}$ & $\begin{array}{c}\mathrm{L}_{\mathrm{c}} \\
(\mathrm{nm})\end{array}$ & $\begin{array}{c}\mathrm{d}_{002} \\
(\mathrm{~nm})\end{array}$ & $\begin{array}{c}\mathrm{L}_{\mathrm{c}} \\
(\mathrm{nm})\end{array}$ & $\begin{array}{c}\mathrm{d}_{002} \\
(\mathrm{~nm})\end{array}$ & $\begin{array}{c}\mathrm{L}_{c} \\
(\mathrm{~nm})\end{array}$ & $\begin{array}{c}\mathrm{L}_{a} \\
(\mathrm{~nm})\end{array}$ \\
\hline GX-10 & 0.338 & 14.1 & - & - & 0.350 & 2.1 & 7.0 \\
GX-100 & 0.338 & 14.1 & - & - & 0.350 & 2.0 & 8.2 \\
GX-350 & 0.337 & 26.6 & 0.341 & 8.7 & 0.352 & 1.7 & 7.6 \\
GX-700 & 0.337 & 35.5 & 0.340 & 11.4 & 0.349 & 2.1 & 7.2 \\
GX-1000 & 0.336 & 38.4 & 0.339 & 10.0 & 0.350 & 2.0 & 7.1 \\
GX-3000 & 0.336 & 40.3 & 0.340 & 10.7 & 0.349 & 2.1 & 7.2 \\
\hline KS75 & 0.336 & 51.6 & - & - & - & - & $>100$ \\
Glassy carbon & 0.338 & 14.1 & - & - & 0.357 & 1.5 & 6.8 \\
\hline
\end{tabular}

${ }^{\text {a }}$ Commercial graphite 


\section{FIGURE CAPTIONS}

FIGURE 1. Pore size distributions of the CXs and GXs as measured by $\mathrm{Hg}$ intrusion porosimetry.

FIGURE 2. XRD profiles of the different GXs.

FIGURE 3. Semi-quantitative analysis of the different carbon contributions to the (002) XRD profiles of the different GXs: a) particulate; b) $\mathrm{GXs}<25 \mu \mathrm{m}$.

FIGURE 4. Evolution with $G X$ average pore size of the $L_{c}$ crystal sizes corresponding to the graphitic, turbostratic and non-graphitic carbon contributions to the (002) XRD profiles of the GXs. Filled symbols correspond to particulate GXs. Void symbols correspond to $\mathrm{GXs}<25 \mu \mathrm{m}$.

FIGURE 5. SEM images of the CXs: a) CX-10; b) CX-100; c) CX-350; d) CX-700; e) CX-1000; f) CX-3000.

FIGURE 6. a) (002) XRD profiles of the GXs<25 $\mu \mathrm{m}$; b) comparison between the (10) and (004) XRD profiles of particulate and <25 $\mu \mathrm{m}$ GXs.

FIGURE 7. TEM images representative of amorphous carbon areas on GXs: a) GX-1000; b) GX-100.

FIGURE 8. TEM images representative of areas showing parallel graphene stacking on GXs: a) GX-10; b), c) and d) GX-1000; e) GX-3000<25 $\mu \mathrm{m}$.

FIGURE 9. SEM micrographs showing carbon whiskers in: a) GX-100; b) GX-3000<25 $\mu \mathrm{m}$.

FIGURE 10. TEM images of carbon whiskers in GX-3000<25 $\mu \mathrm{m}$ : a) carbon whisker; b) carbon whisker nanostructure; c) higher magnification detail of $b)$.

FIGURE 11. Representative Raman spectra of particulate GXs: a) spectrum obtained on powdered GX-1000; b) spectrum obtained on the outermost surface of GX-10 particles. 
FIGURE 12. Electrical conductivity values measured on pellets of CXs and GXs, using a FPP technique. 


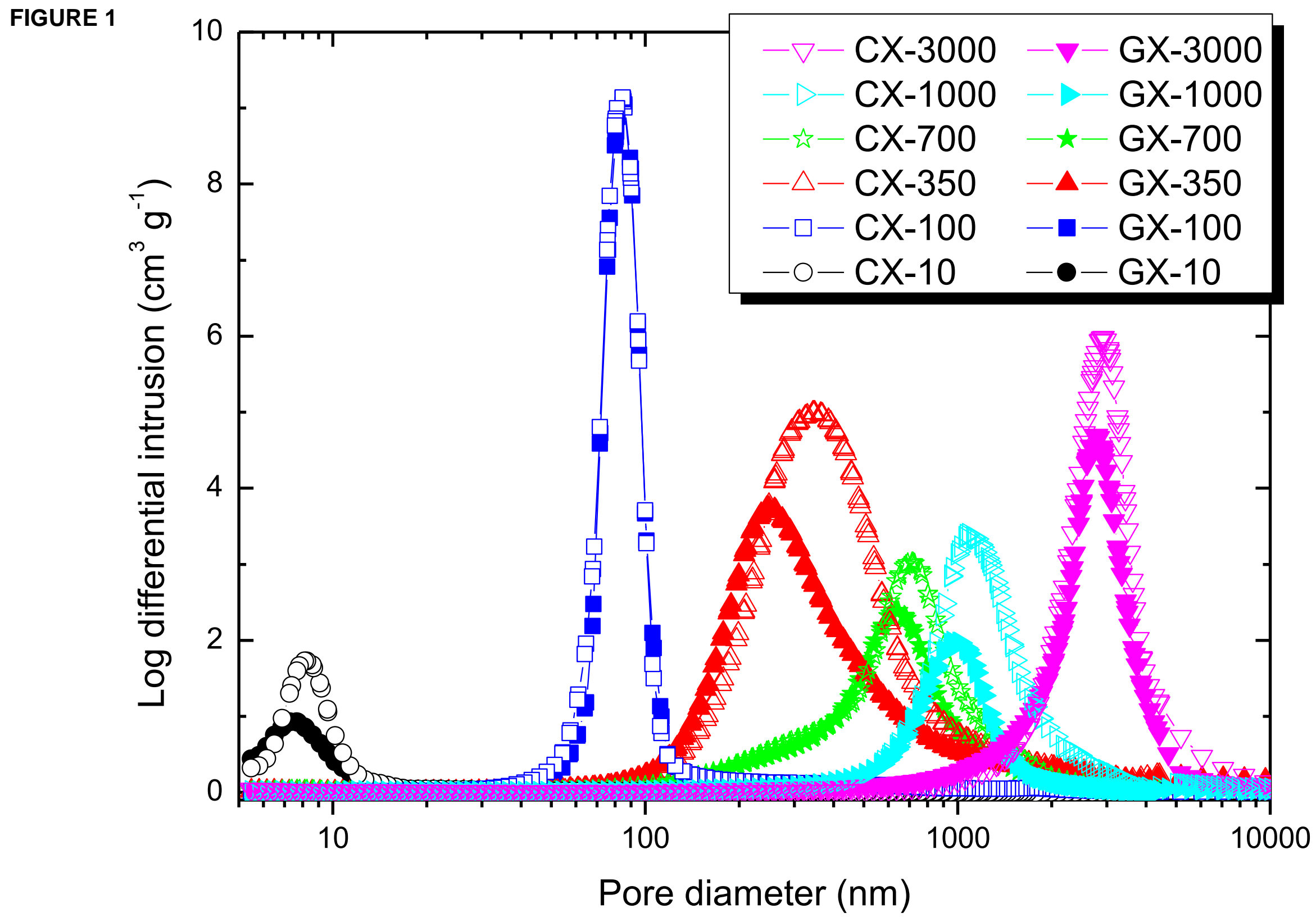


FIGURE 2a.

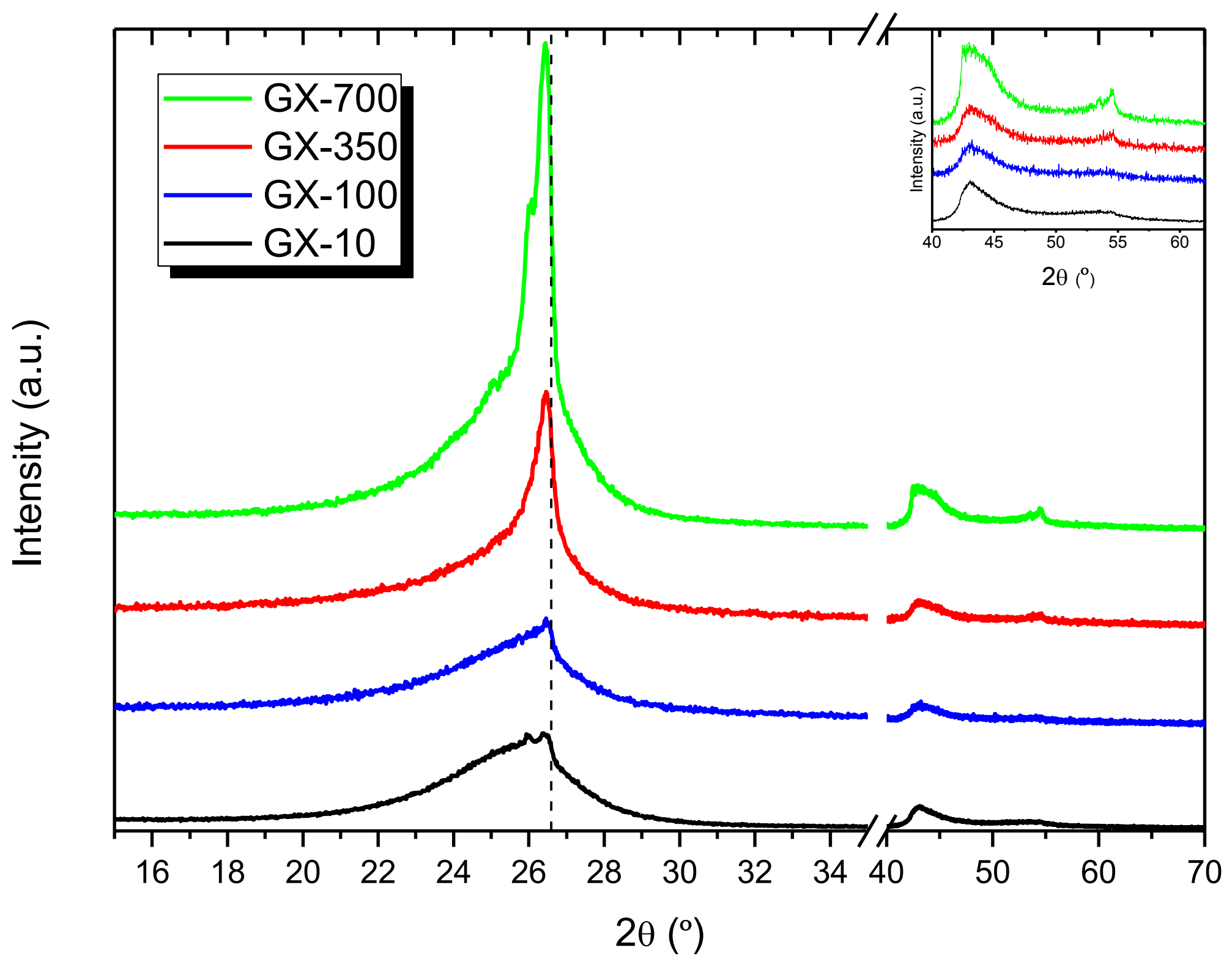


FIGURE $2 b$.

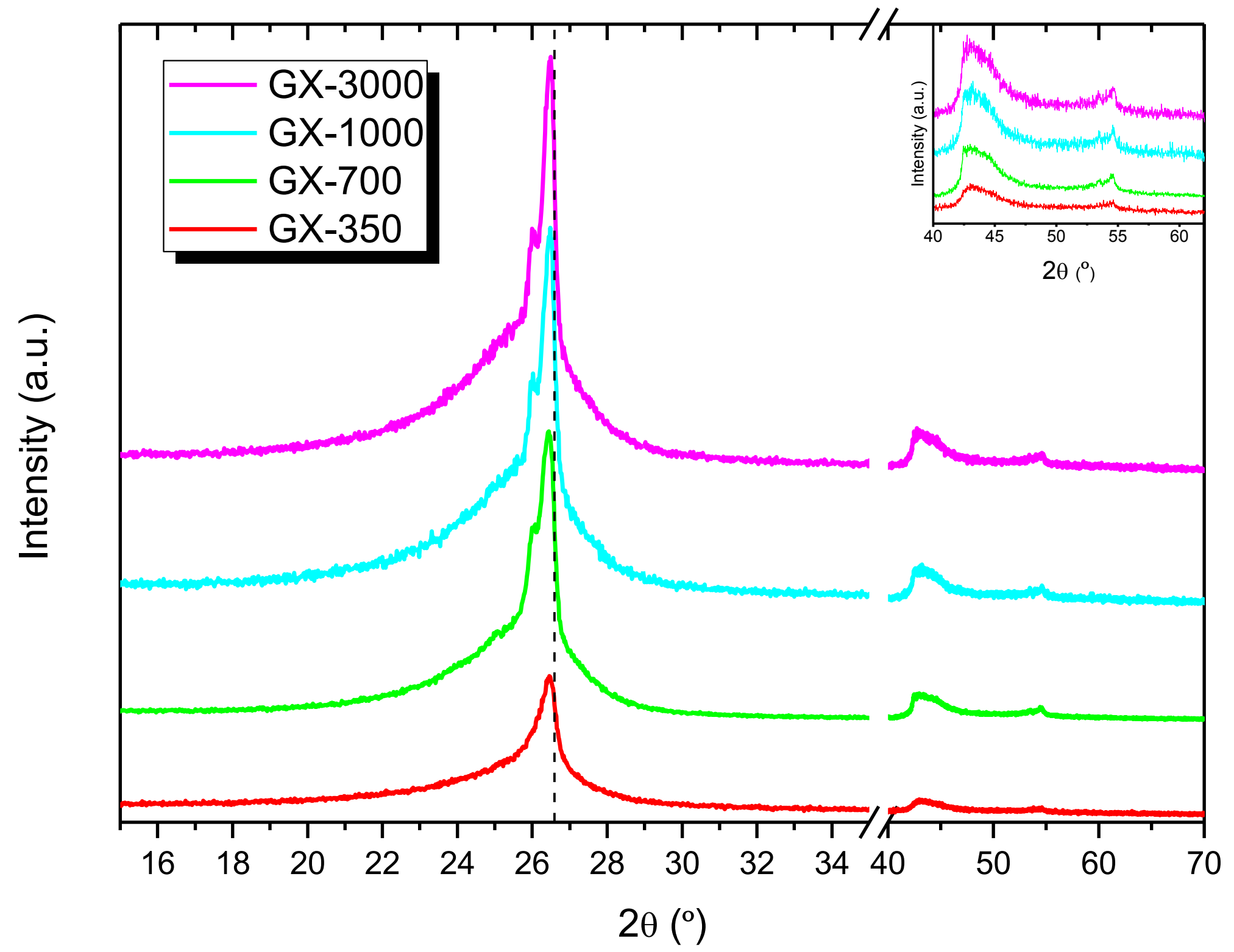




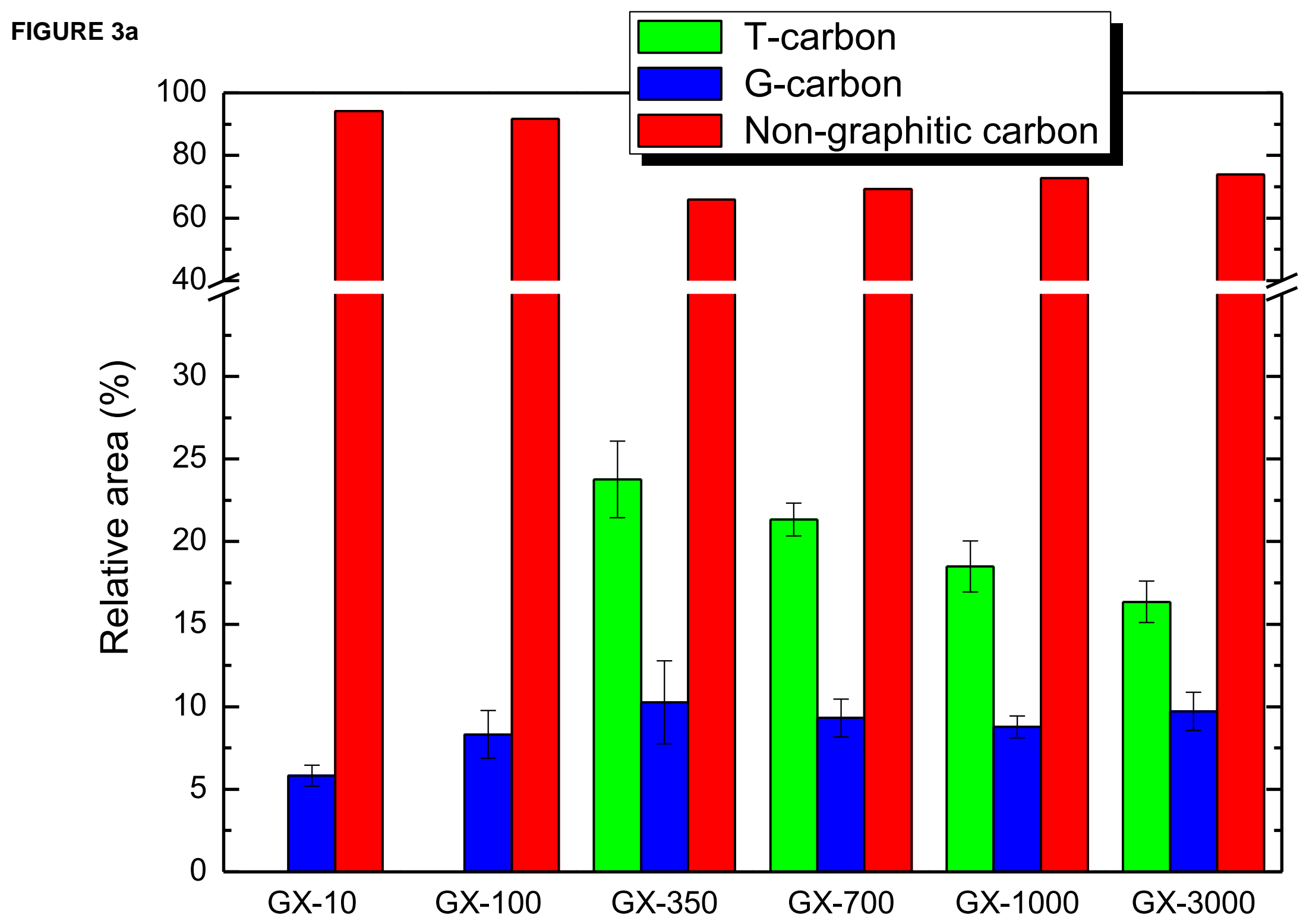


FIGURE 3b

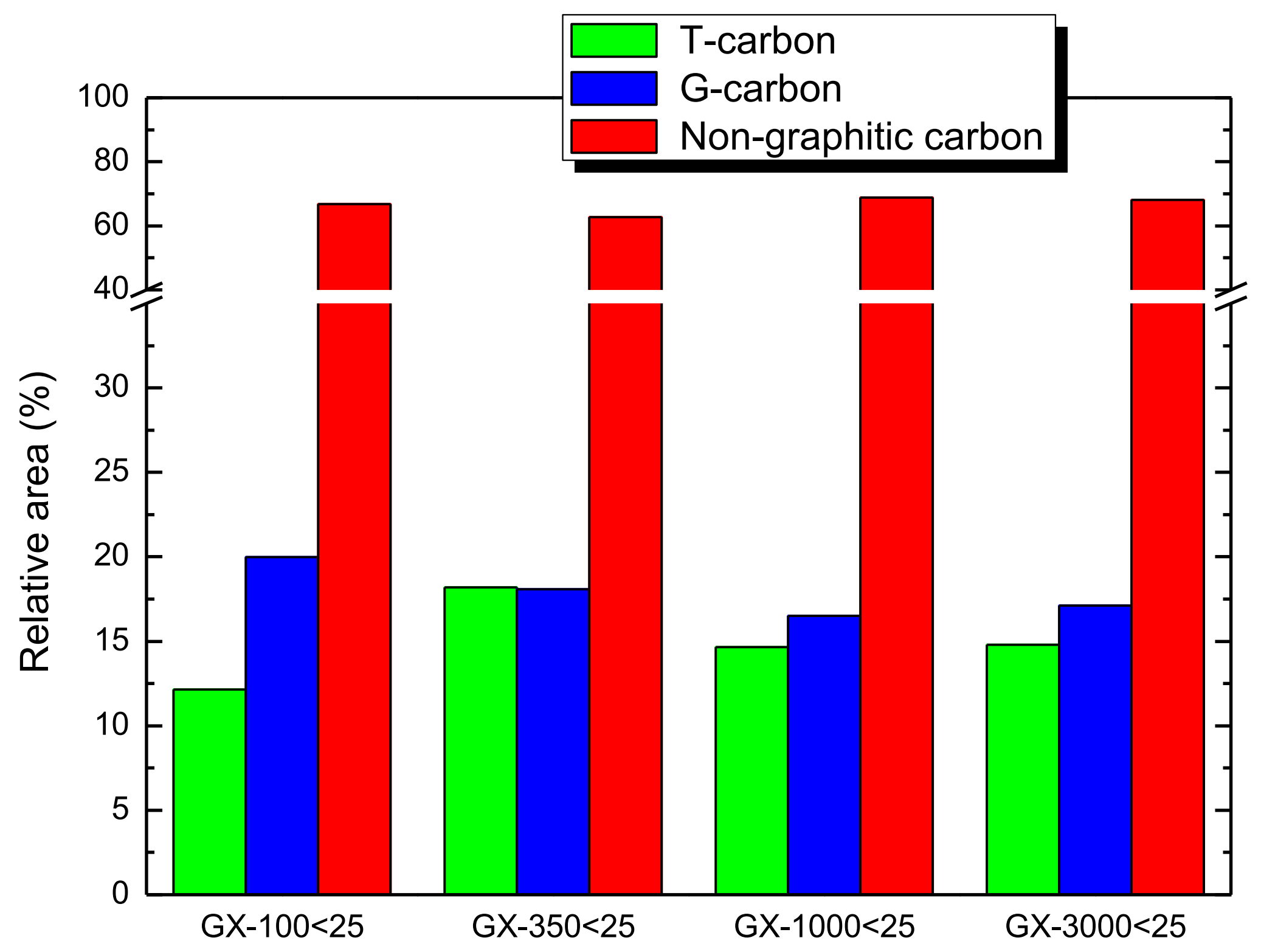




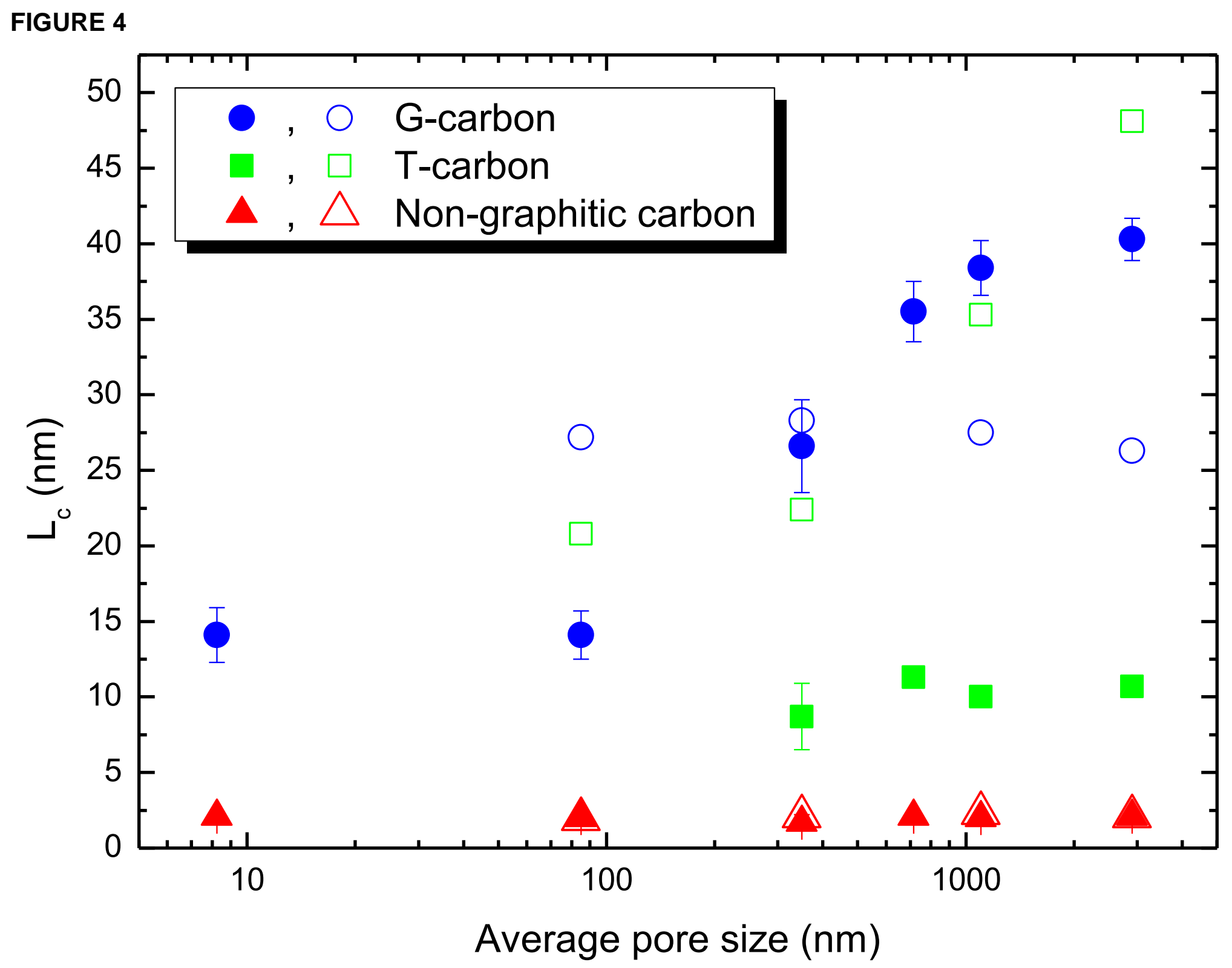


FIGURE 5

FIGURE 6a

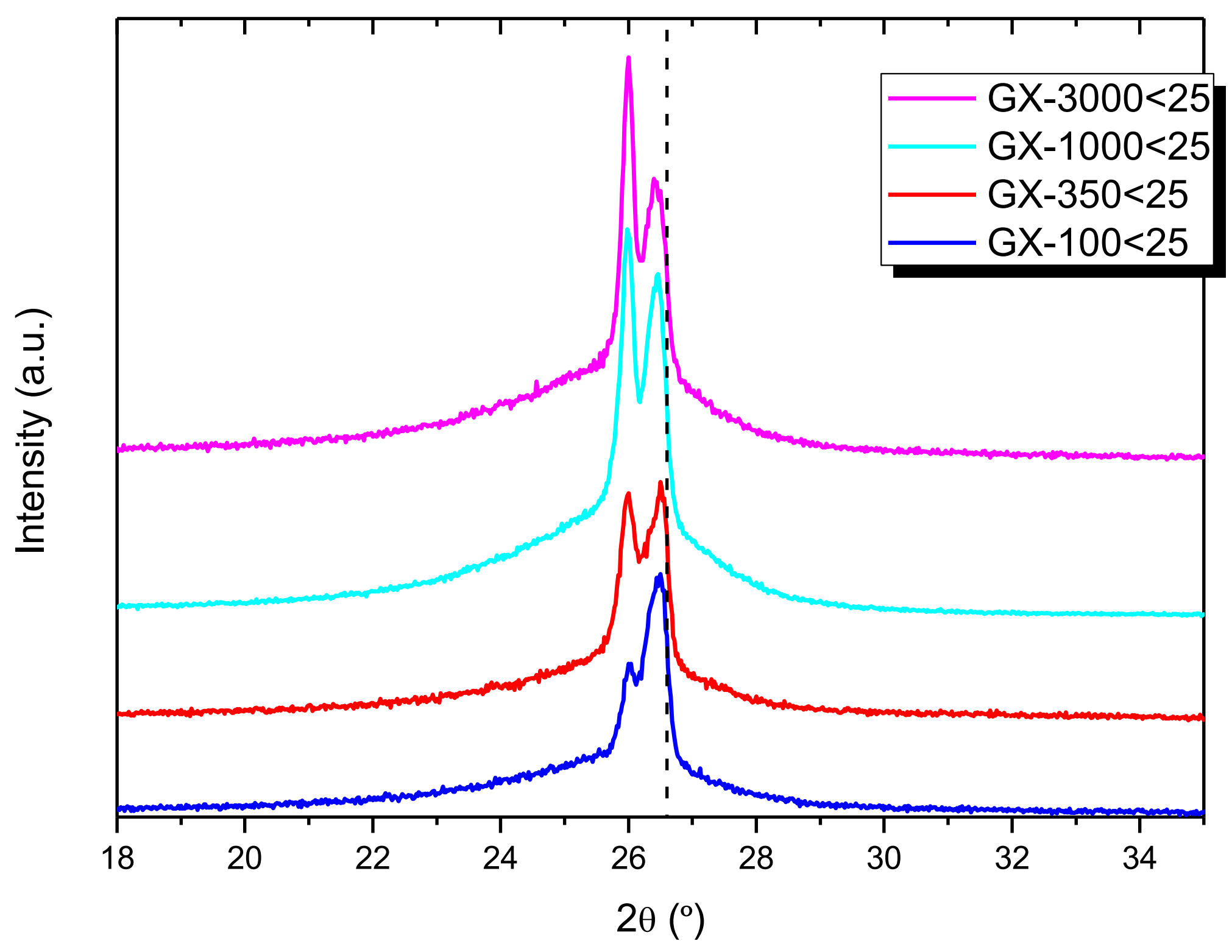


FIGURE 6b




FIGURE 7
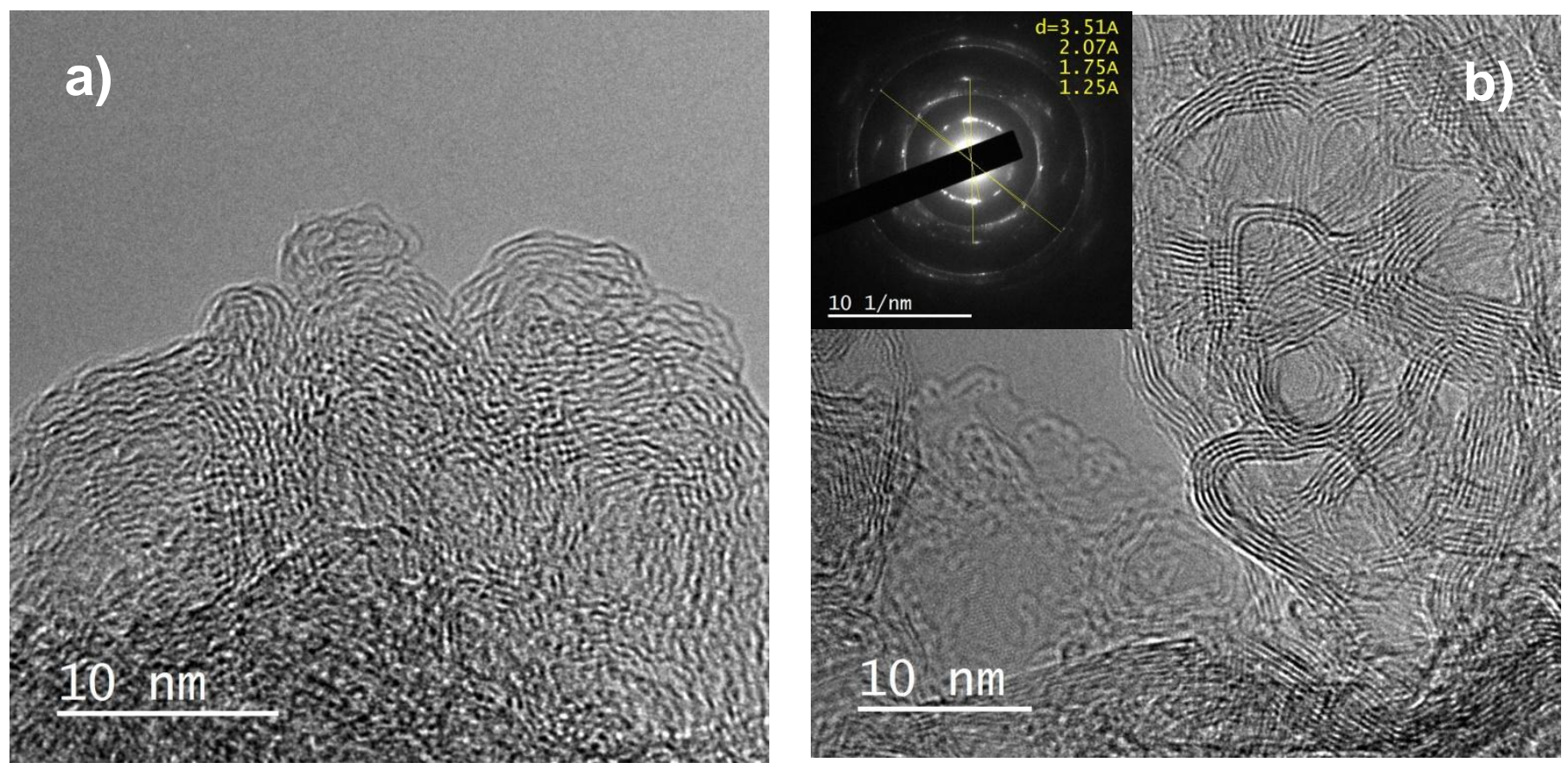
FIGURE 8
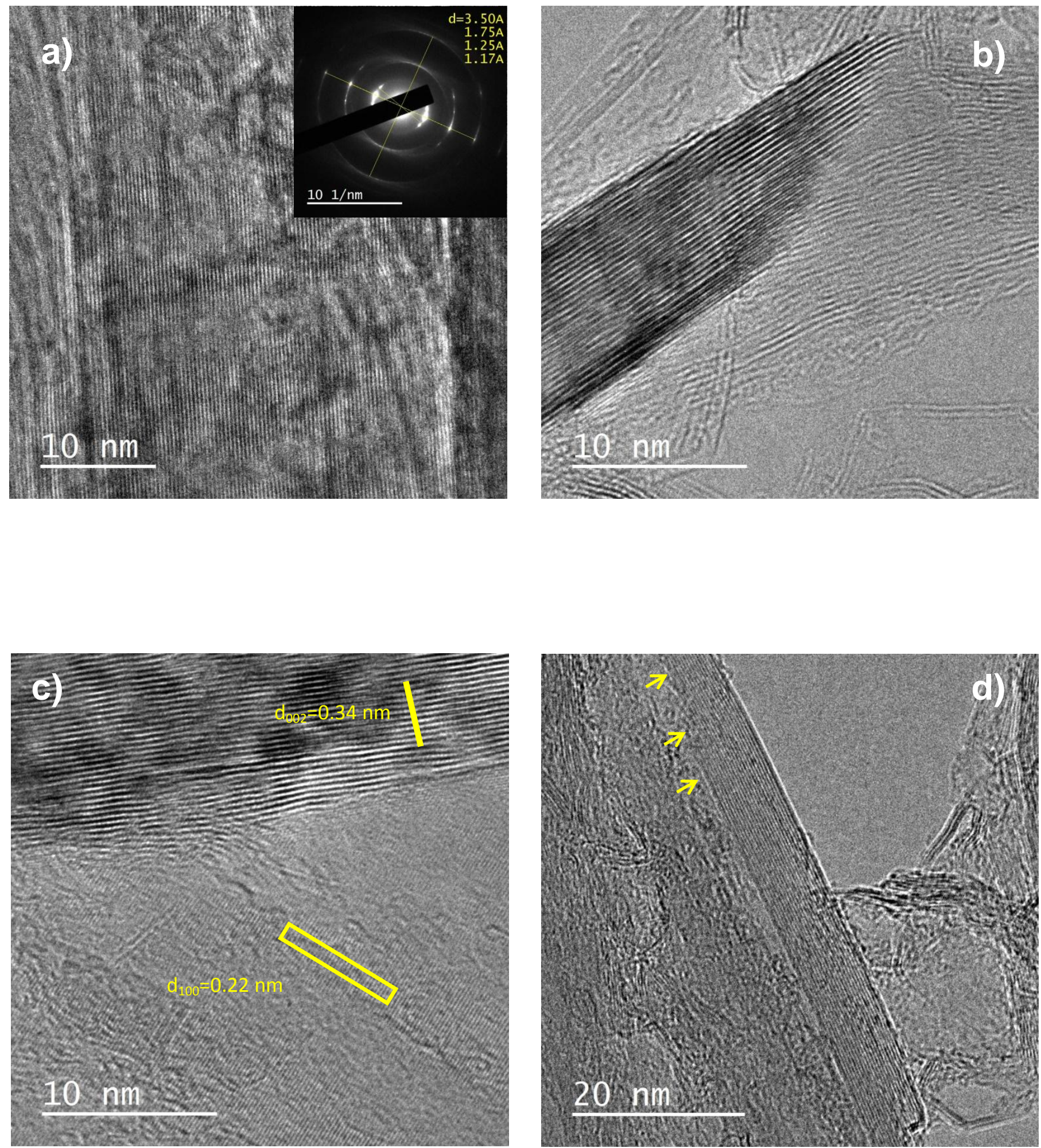
FIGURE 8 (cont.)

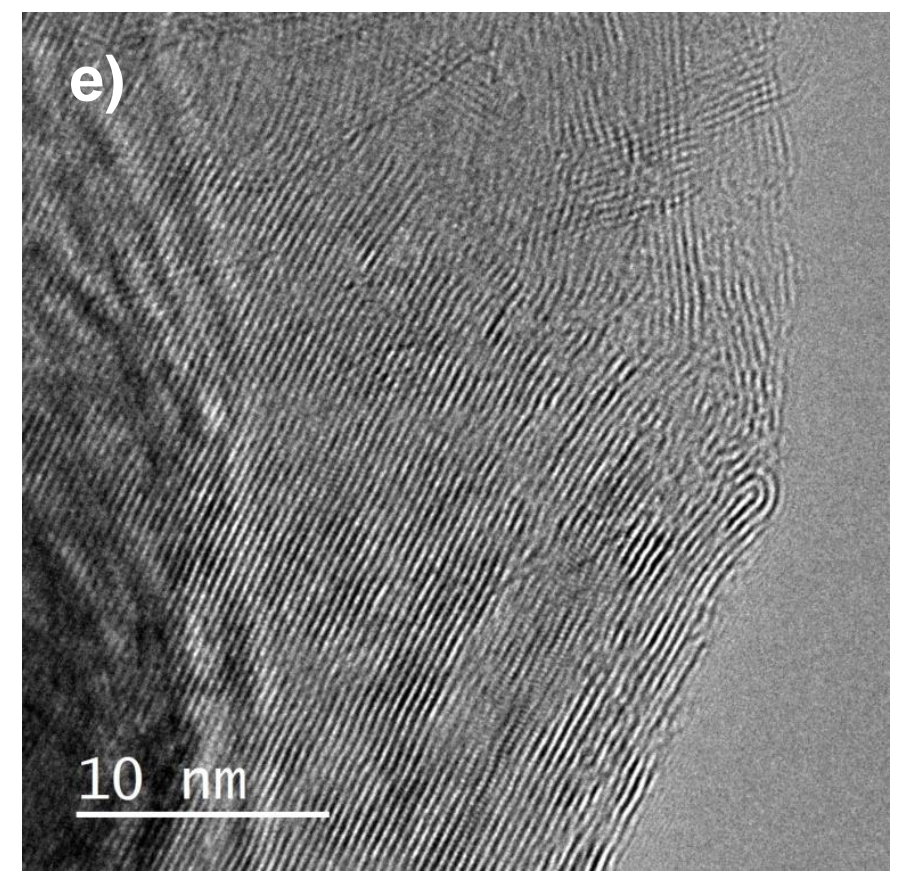


FIGURE 9
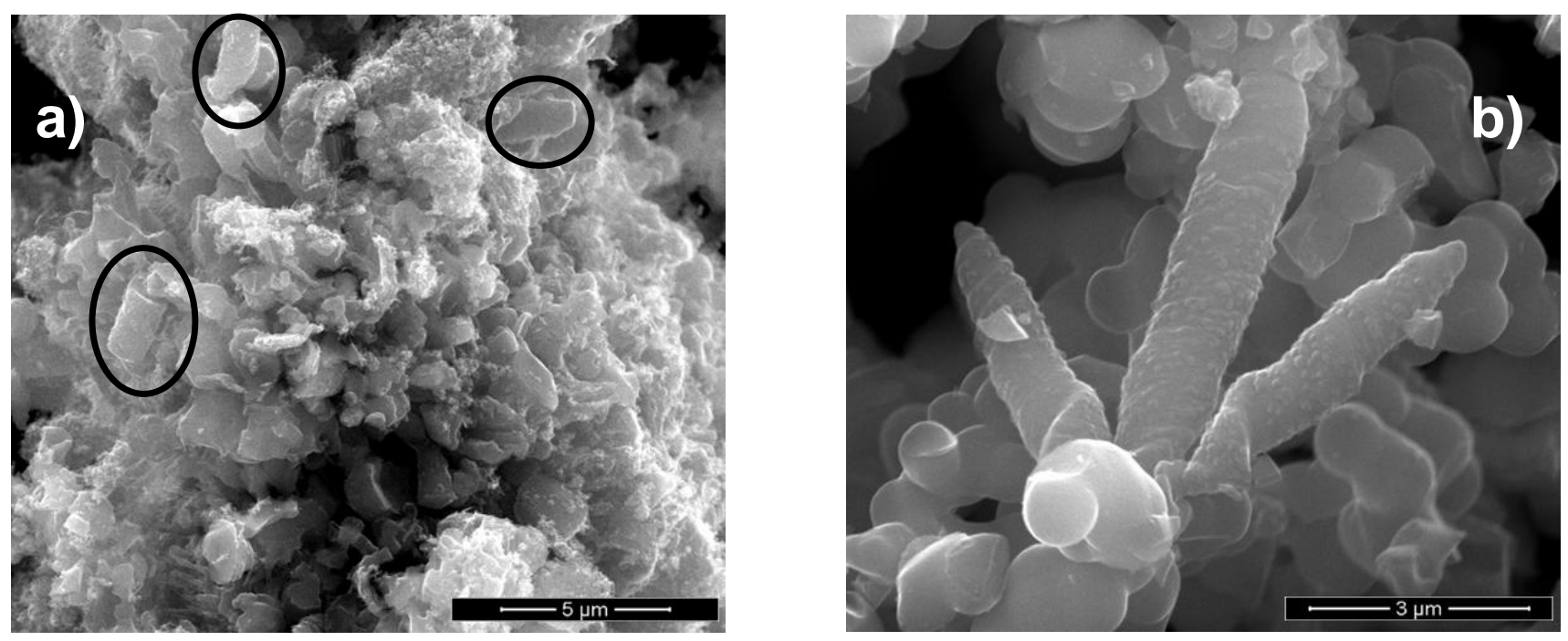
FIGURE 10
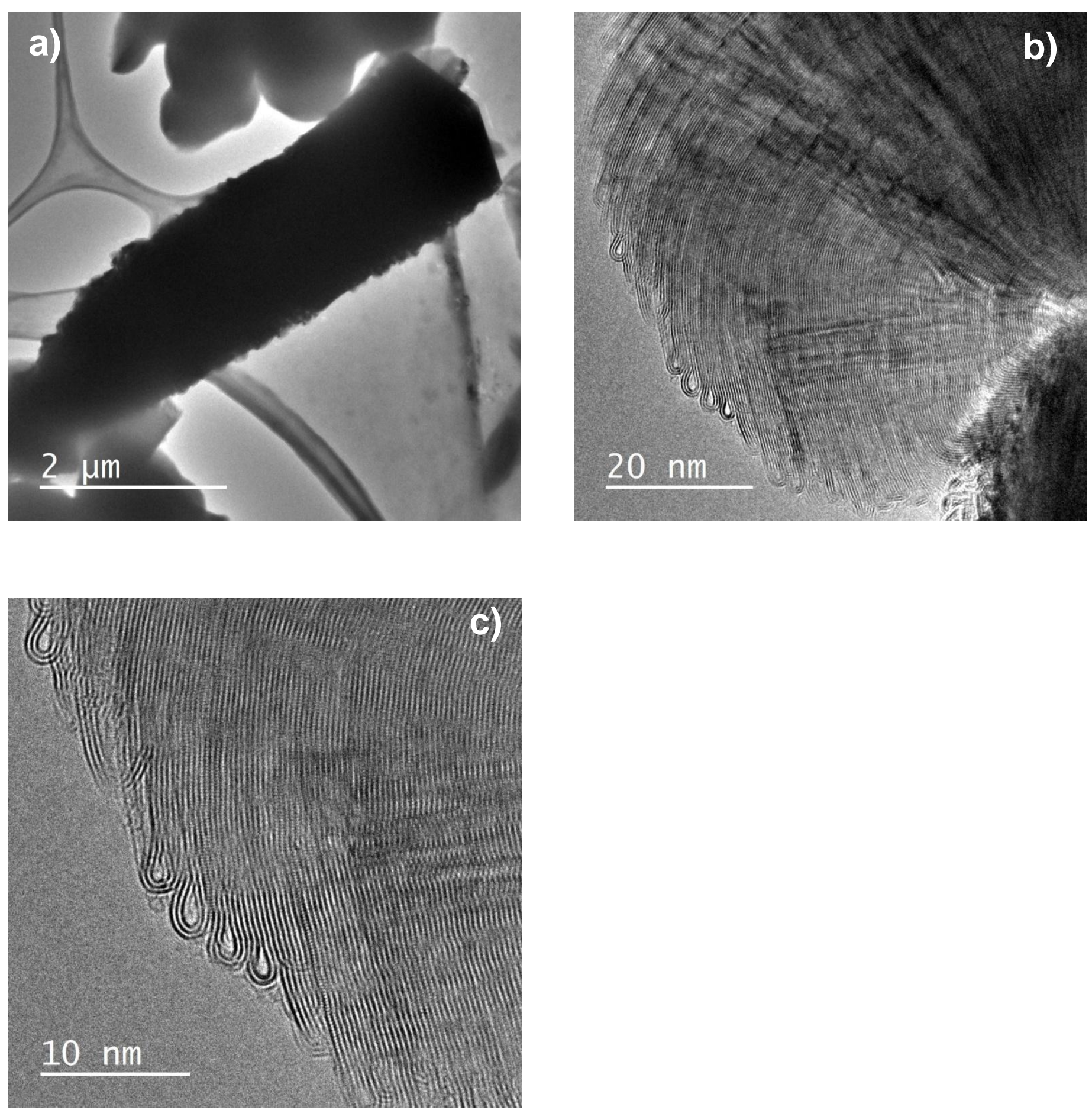


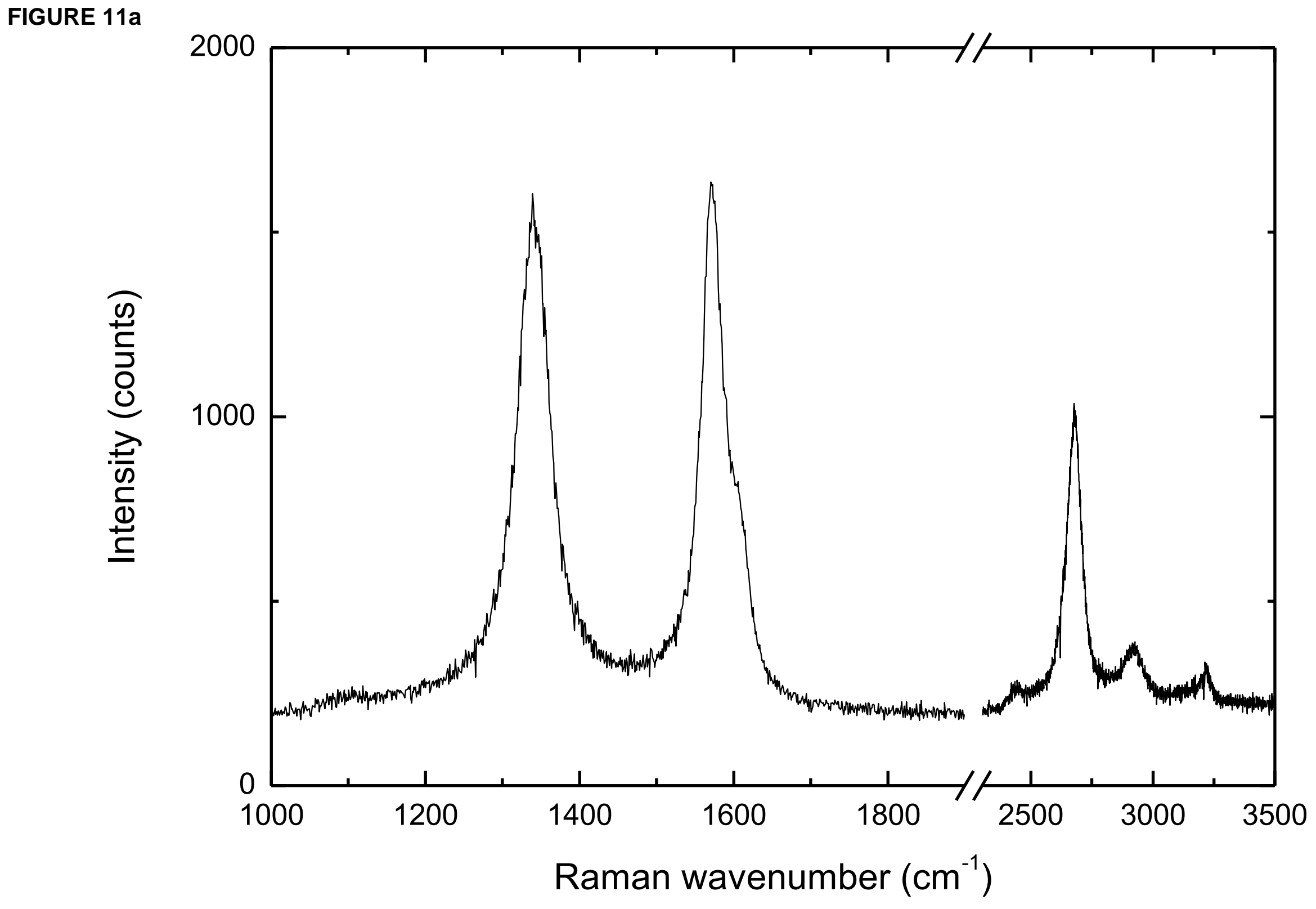




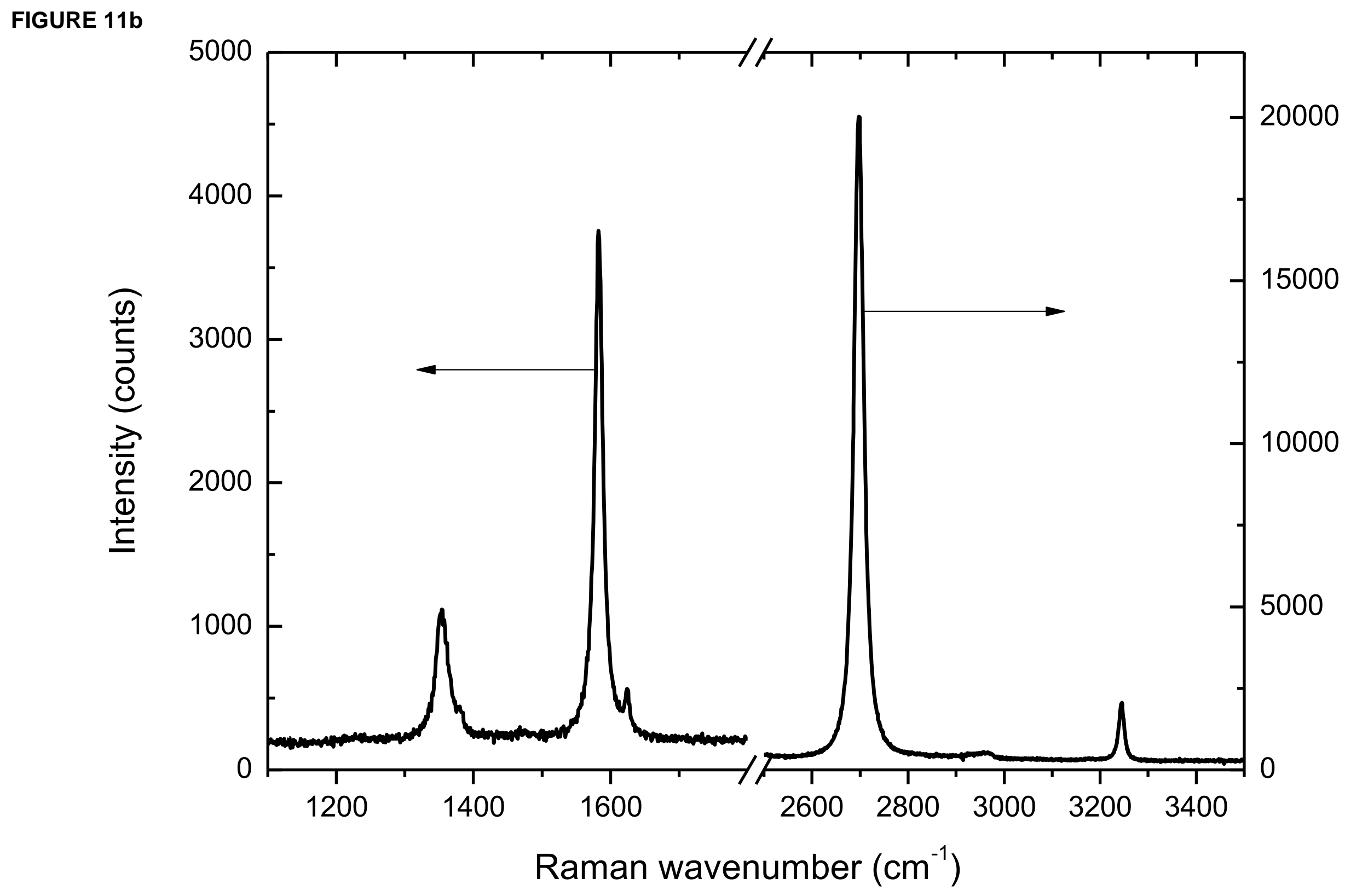




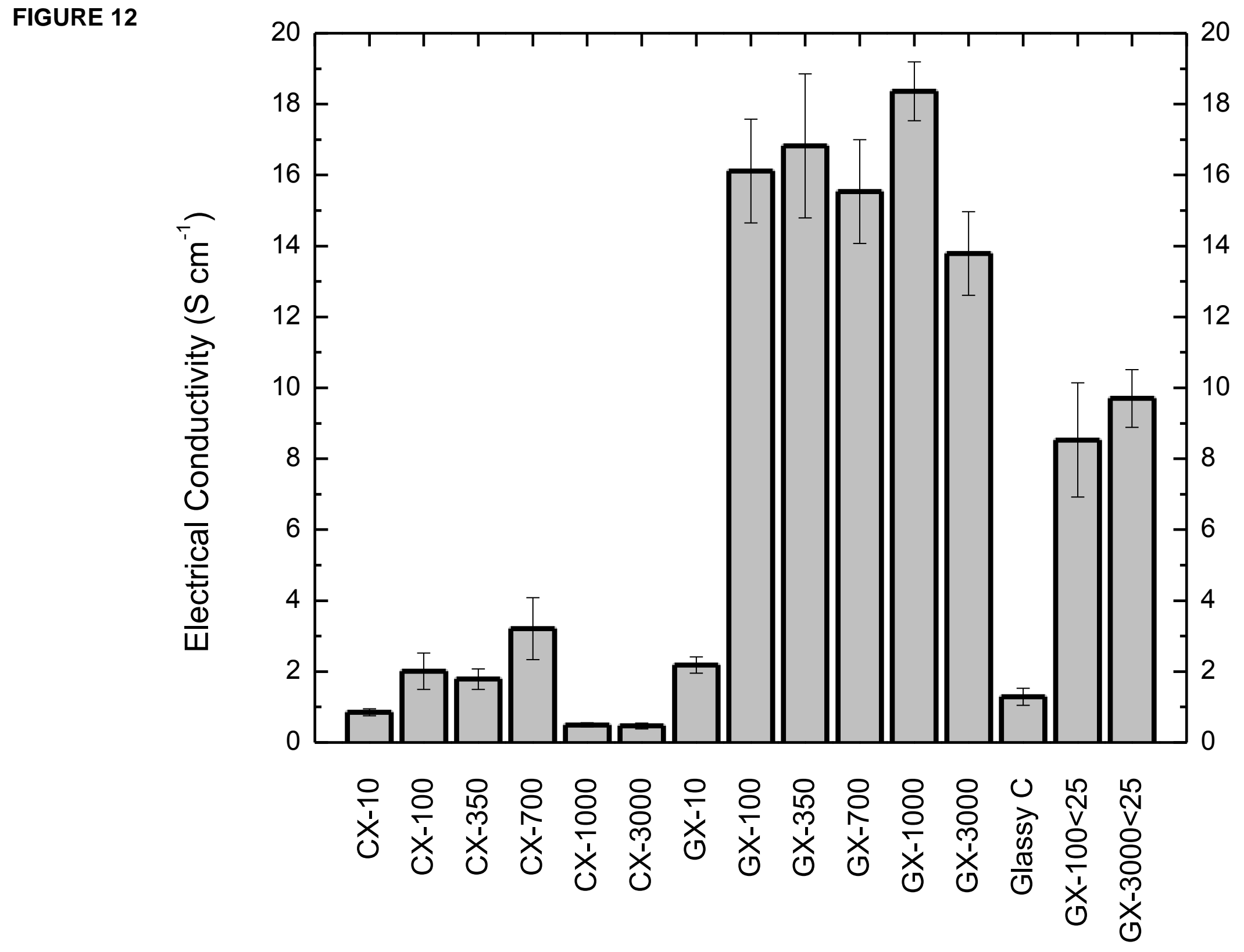

